Revue Française de Civilisation Britannique

XVII-2 | 2012

Minorités, intégration en Grande-Bretagne et dans les pays du Commonwealth

\title{
Identités nationales, identités « raciales » en Océanie
}

National Identities and 'Racial' Identities in the Pacific

\section{Adrien Rodd}

\section{CpenEdition}

Journals

Édition électronique

URL : http://journals.openedition.org/rfcb/679

DOI : $10.4000 /$ rfcb. 679

ISSN : 2429-4373

Éditeur

CRECIB - Centre de recherche et d'études en civilisation britannique

Édition imprimée

Date de publication : 15 octobre 2012

Pagination : 113-142

ISBN : 2-911580-37-0

ISSN : 0248-9015

Référence électronique

Adrien Rodd, «Identités nationales, identités « raciales » en Océanie », Revue Française de Civilisation Britannique [En ligne], XVII-2 | 2012, mis en ligne le 15 mars 2016, consulté le 20 avril 2019. URL http://journals.openedition.org/rfcb/679; DOI : 10.4000/rfcb.679

Ce document a été généré automatiquement le 20 avril 2019

\section{c) ()ㅇㅇ}

Revue française de civilisation britannique est mis à disposition selon les termes de la licence Creative Commons Attribution - Pas d'Utilisation Commerciale - Pas de Modification 4.0 International. 


\title{
Identités nationales, identités « raciales » en Océanie
}

\author{
National Identities and 'Racial' Identities in the Pacific
}

\section{Adrien Rodd}

1 La construction identitaire nationale a été, à des degrés plus ou moins importants, une préoccupation dans les États issus de la décolonisation dans le Pacifique, à l'instar d'autres régions du monde. Comme ailleurs, la volonté de bâtir un sentiment d'unité, d'appartenance commune à la nation sur le fondement de critères aisément identifiables, a parfois paru difficilement compatible avec la reconnaissance de la diversité sous toutes ses formes, et avec l'acceptation des minorités au sein de l'imaginaire nationalnotamment les minorités rendues particulièrement visibles par leur phénotype « étranger ». Cet article s'intéresse aux États nés des anciennes colonies britanniques en Océanie. Cet ensemble recouvre des pays en apparence bien différents - des colonies de peuplement que furent l'Australie et la Nouvelle-Zélande, aux îles du Pacifique demeurées (à deux exceptions près) majoritairement autochtones. Pourtant, malgré leurs différences, notamment en termes de population, plusieurs de ces pays ont connu ou connaissent la tentation de fonder leur affirmation d'unité identitaire nationale sur une adéquation entre la nation et son ethnie, voire sa 'race', majoritaire. Dans un tel contexte, la place des minorités s'est inscrite dans une gradation entre pleine jouissance des droits civiques malgré une exclusion, explicite ou non, de l'imaginaire national ; et un rejet total prônant une pleine homogénéité « raciale » de la société nationale.

\section{Des terres pour la « race » blanche}

2 L'histoire de la Nouvelle-Zélande et de l'Australie les distingue dans une grande mesure des autres pays de la région. L'Australie, on le sait, n'avait pas pour vocation première d'être une colonie florissante, accueillant en grand nombre des migrants britanniques venus de leur plein gré. Le statut de colonies de peuplement de ces deux territoires fut en partie une réponse à des initiatives privées. Ce fut un homme politique et homme d'affaires anglais, Edward Gibbon Wakefield, qui, le premier, orchestra de sa propre 
initiative un début de peuplement organisé de l'Australie méridionale, dans les années 1830. Il fonda ensuite la New Zealand Company, anticipant sur - voire précipitant l'annexion britannique de la Nouvelle-Zélande en y dépêchant des colons en $1839^{1}$.

Dès lors, ces territoires aux antipodes n'étaient viables que dans la mesure où ils accueilleraient des colons en grand nombre, agents de leur transformation en sociétés ordonnées, inspirées du modèle britannique. À partir de la fin du XIX ${ }^{e}$ siècle, une immigration blanche massive était également considérée comme une nécessité absolue pour la sécurité nationale, face au fantasme d'un "péril jaune», militaire ou démographique. Le slogan alarmiste «se peupler ou périr " (populate or perish) provint du premier ministre australien Billy Hughes en $1937^{2}$, résumant une notion clef des discours politiques depuis des décennies. De 1851 à 1861, $40 \%$ des immigrés arrivant en Australie avaient bénéficié de financement public pour permettre leur voyage. Ce taux atteignit plus de $50 \%$ entre 1861 et 1900 , puis plus de $67 \%$ de 1919 à $1929^{3}$. Ces financements s'adressaient en priorité aux ressortissants des Îles britanniques - dont les Irlandais, qui furent nombreux à en bénéficier. Quant à la Nouvelle-Zélande, dans les années 1870, la politique du premier ministre Julius Vogel résulta en un bond démographique. Il finança avec des fonds publics la venue d'une centaine de milliers d'immigrés blancs, originaires à plus de $90 \%$ des îles britanniques ${ }^{4}$.

Initialement, les politiques d'immigration visaient avant tout à faciliter et à encourager la venue de migrants britanniques, dans le but de développer la société coloniale et de la consolider, tant face aux autochtones qu'aux «hordes» asiatiques perçues comme une menace bien proche. Mais à partir de la fin du XIXe siècle, ces politiques d'incitation allaient s'accompagner de mesures restrictives fermant ces deux pays aux migrants nonblancs. En 1879, Sir George Grey, Premier ministre de Nouvelle-Zélande, affirma devant la Chambre des Représentants de la colonie la nécessité pour ses habitants « d'être fidèles à eux-mêmes, et de préserver, indemne et sans mélange, cette population anglo-saxonne qui l'habite à présent $»^{5}$. Bien plus tard, en 1924, l'un de ses successeurs, le premier ministre William Massey, écrivit qu'il s'évertuait à faire du pays « un foyer digne des meilleurs éléments de la race anglo-saxonne - un pays de l'Homme blanc $»^{6}$. Au même moment, un journaliste australien, Keith Murdoch, écrivait: «[L] a politique [australienne] est fondée [...] sur un des idéaux les plus nobles qui puissent motiver l'homme. [...] La pureté raciale est son objet sacré, bien plus sacré pour la nouvelle génération d'Australiens que toute autre attache terrestre ${ }^{7} »$. Le Haut-commissaire australien au Royaume-Uni affirmait pour sa part, dans une lettre au Times, que l'adoption des politiques de l'Australie blanche avait eu lieu «parce que la possibilité d'un continent à la race pure était devenue une question de fierté nationale unanime. Elle l'est toujours, et elle le demeurera ${ }^{8} »$. Entre les deux dates, le discours n'avait pas varié ; cette idée de "pays de l'Homme blanc ", de terre réservée à la 'race anglo-saxonne', demeura consensuelle pour les gouvernements successifs dans les deux colonies. Alfred Deakin, l'une des personnalités politiques les plus influentes au moment de la fédération de l'Australie en 1901, affirmait que les Australiens devaient être " un seul peuple, et demeurer un seul peuple, sans mélange avec d'autres races", car "[l]'unité de l'Australie n'est rien si cela n'implique pas une race unie ${ }^{9}$ ». "Pays », " peuple » et "race » devenaient essentiellement synonymes dans le contexte australien et néo-zélandais.

Principale minorité visible dans les mines d'or, à partir des années 1850, les Chinois furent la cible de préjugés sinophobes, qui aboutirent aux premières politiques d'exclusion. Le rejet qu'ils subirent était motivé principalement par quatre facteurs. Les 
travailleurs, les dirigeants syndicaux et les journalistes évoquaient la menace qu'ils feraient peser sur l'emploi des Blancs, tandis que les hommes politiques et les théoriciens de la «race »s'inquiétèrent d'une "contamination» de la « race blanche», risquant de perdre sa « pureté »" ${ }^{10}$. Cette " contamination » apparaissait d'autant plus évidente que les Chinois furent caricaturés en hommes fourbes, pervers, drogués à l'opium - une menace pour les femmes blanches. Le New Zealand Times en 1896 titrait : " Le Quartier chinois de Wellington : des lieux de vice asiatique comme la peste parmi nous ${ }^{11}$. Rapidement, le potentiel démographique de la Chine fut aussi décrit comme un danger pour la sécurité nationale des "petites» colonies blanches - le «Péril jaune». Enfin - et paradoxalement-, les Chinois furent accusés de ne pas s'investir dans l'avenir de la colonie. Ces migrants temporaires (huaqiao) ne cherchaient qu'à s'enrichir avant de retourner en Chine, firent remarquer les hommes politiques néo-zélandais des années 1890. Parlant souvent mal l'anglais, de religion "païenne », ils furent décrits comme inassimilables, ne s'identifiant aucunement à leur pays de résidence ${ }^{12}$. Ils étaient donc des étrangers permanents - une anomalie dans des sociétés qui se voulaient homogènes.

6 À partir des années 1880 , les autorités australiennes et néo-zélandaises commencèrent à interdire aux Chinois l'accès à leur territoire, le plus souvent encore de manière ponctuelle et aléatoire, pour satisfaire aux revendications populaires. Cette époque correspondait, en Nouvelle-Zélande, à une stagnation économique, propice à la xénophobie. En 1881, la loi Chinese Immigrants Act, en Nouvelle-Zélande, imposa aux seuls Chinois le paiement d'une taxe d'entrée de $£ 10$, et interdit à tout navire d'aborder dans ses ports avec à son bord plus d'un migrant chinois par centaine de tonnes de marchandises. En 1896, la loi Asiatic Restriction Bill stipula qu'aucun navire ne pourrait transporter plus d'un migrant chinois pour deux cent tonnes de marchandises, et tout immigré chinois - défini par sa "race", et non par sa nationalité - devrait s'acquitter d'une taxe d'entrée de $£ 100$ - une somme astronomique pour l'époque. En 1899, la Nouvelle-Zélande adopta la loi Immigration Restriction Act. Celle-ci fermait les frontières de la nation insulaire à toute personne n'étant pas de descendance britannique ou irlandaise et qui échouerait à un exercice de dictée en langue anglaise - cette dernière étant une idée empruntée à la colonie sud-africaine du Natal. Le nombre de Chinois en NouvelleZélande fut réduit de 5004 en 1891 à 2857 en 1901, puis à 2072 en $1916^{13}$. La "politique de la Nouvelle-Zélande blanche" (White New Zealand policy) s'était dotée d'un appareil législatif efficace. Les auteurs du recensement de 1921 pouvaient ainsi déclarer :

Du point de vue de la race, la population du Dominion est, et a toujours été, conforme à une haute norme de pureté; en effet, le maintien d'une norme de population purement européenne ou "blanche" a été invariablement pris en compte dans la législation sur l'immigration. L'importance de la pureté raciale est reconnue depuis longtemps. L'histoire nous a montré que le mélange de la race blanche et des races dites de couleur n'est pas favorable à l'amélioration des types raciaux $^{14}$.

7 La préservation de la "pureté raciale » était donc une fin en soi ; toute présence nonblanche - outre celle des Maori - risquait d'entraîner une " pollution », et un « déclin » du «type racial » blanc, le plus «avancé ». La position de l'Australie était similaire, et le gouvernement australien en 1925 publia un communiqué se félicitant du fait que sa population était «britannique » à « $99 \%$ \% - et qu'elle le resterait ${ }^{15}$. Ce chiffre excluait bien sûr les Aborigènes, qui n'étaient pas comptés dans les recensements. Le recensement de 1947 annonça que la population non-blanche de l'Australie était de $0,25 \%^{16}$. Tous les 
Blancs n'étaient pas 'britanniques', mais tous les «Britanniques » étaient blancs, et comprenaient officiellement la quasi-totalité de la population. De même, en NouvelleZélande, des hommes politiques à la fin du XIX siècle et au début du XXe affirmèrent à plusieurs reprises que le pays était britannique à «98,5\%» - affirmation qui requérait "[d] ivers tours de passe-passe statistiques et sémantiques ${ }^{17}$ \#. Les statistiques officielles furent invoquées pour preuve de l'unité « raciale », et donc nationale, de chaque pays.

Dès l'unification de l'Australie en janvier 1901, le gouvernement œuvra à la consolidation d'une société blanche. Le Parlement fédéral adopta la loi Pacific Island Labourers Act - six jours avant d'adopter la loi Immigration Restriction Act. Ces deux textes, combinés au Commonwealth Franchise Act de 1902, qui interdisait le droit de vote à toute personne nonblanche, constituèrent les fondements de la "politique de l'Australie blanche " (White Australia policy). Le Pacific Island Labourers Act prévoyait la déportation massive des travailleurs mélanésiens présents dans le nord du pays. Au nombre de 10000 en 1901, ils n'étaient plus que 4500 en 1906, et seuls 1654 furent autorisés à rester après $1908^{18}$. Ayant « réglé » le "problème » des Mélanésiens, les législateurs se tournèrent vers les Chinois. Il y avait alors environ 30000 personnes nées en Chine résidant en Australie ${ }^{19}$. La jeune nation, unie pour la première fois et en passe de se définir, fit de la "politique blanche» l'une de ses priorités. Ainsi, «l'exclusion [...] d'Asiatiques fut au centre de la formation même de la nation australienne moderne $»^{20}$; l'Australie, à sa naissance, fut définie explicitement comme un pays blanc. Restreindre l'immigration chinoise n'était pas une idée nouvelle. Des chercheurs d'or blancs dans les années 1850 avaient demandé l'interdiction de leur entrée sur le territoire. Dans les années 1850, 1860 et 1870, il y eut plusieurs violentes émeutes anti-chinoises, notamment dans les mines d'or, faisant plusieurs morts ${ }^{21}$. Dès 1855 , les autorités du Victoria leur imposèrent une taxe d'entrée de $£ 10^{22}$. En 1884, l'Assemblée législative du Queensland adopta une loi interdisant, comme en Nouvelle-Zélande, à tout navire d'« importer» en Australie plus d'un migrant chinois pour cinquante tonnes de marchandises, et imposant à ces immigrés une taxe de $£ 20^{23}$. La Nouvelle-Galles du Sud et le Victoria firent de même quelques années plus tard.

La loi fédérale Immigration Restriction Act de 1901 s'inspira du précédent néo-zélandais. Les agents d'immigration furent invités à soumettre à un test de dictée, dans n'importe quelle langue européenne, tout candidat non-blanc à l'immigration. Une circulaire transmise par le gouvernement aux autorités douanières précisait explicitement que "le test, lorsqu'il est appliqué à un immigré, est fait pour empêcher absolument cette personne d'entrer en Australie [...]. Le test doit donc être appliqué dans une langue que l'immigré ne connaît pas suffisamment bien pour pouvoir rédiger une dictée dans cette langue ${ }^{24} »$. Un député du parlement du Queensland, interrogé par un journaliste en 1902, confirma en outre que "les douaniers ont reçu pour ordre de n'appliquer cette loi qu'aux Asiatiques ${ }^{25}$ ». La «politique de l'Australie blanche » fut, dans une large mesure, un succès. La population asiatique, notamment chinoise, cessa de croître, et connut un déclin marqué. Au nombre de 29907 en 1901, les personnes nées en Chine et résidant en Australie n'étaient plus que 15224 en 1921, et 6404 en 1947. Parmi eux, il y avait 394 femmes en 1901, 322 en 1911, et 365 en 1921. La population chinoise ne connaîtrait pas d'accroissement naturel conséquent. En 1941, un journaliste pouvait écrire au sujet de la politique de l'Australie blanche que « tous les partis politiques la soutiennent pleinement. Elle est l'une des institutions les plus chéries des Australiens ${ }^{26}$ ».

Quant aux Aborigènes et aux Maori, leur présence était potentiellement problématique pour deux sociétés se voulant exclusivement blanches. Les perceptions à leur égard, et les 
politiques qui en découlèrent, furent néanmoins assez différentes. En Australie, la nature même du projet colonial, qui saisissait les terres aborigènes sans compensation, et déplaçait de force les populations, les contraignant souvent à la famine, ne leur offrait aucune place dans la société blanche. Les massacres furent fréquents, se combinant aux maladies avec des effets dévastateurs. Entre 1788 et 1930, la population aborigène sur l'ensemble de l'Australie chuta d'environ $90 \%^{27}$. Les Maori, pour leur part, devinrent explicitement des sujets britanniques en vertu de l'article trois du Traité de Waitangi ${ }^{28}$. Leur nationalité britannique fut confirmée par la loi Native Rights Act de 1865, qui réaffirmait leur égalité en droits et en devoirs. Aux yeux de la loi, et des autorités, les Maori étaient britanniques. A contrario, les Aborigènes ne disposaient d'aucun statut clairement établi, relatif à leur hypothétique nationalité. Tandis que la Nouvelle-Zélande tentait, de manière souvent imparfaite et discriminatoire, d'encourager la participation des Maori à la vie économique, sociale et politique de la colonie, dont ils étaient citoyens, les Aborigènes «ne furent simplement pas reconnus comme des membres de la société29 ". "Une fois conquis ils souffrirent [...], car ils n'avaient aucune place utile dans la nouvelle société [où] les Aborigènes étaient perçus comme superflus ${ }^{30}$ ». Ils n'eurent pas accès à l'école des Blancs ${ }^{31}$. Les autochtones qui souhaitaient être assimilés ne pouvaient l'être. Dès le XIX ${ }^{e}$ siècle, il fut considéré "désirable d'employer des Maori dans le service public", de les intégrer à l'administration coloniale ${ }^{32}$. Les Pakeha (non-autochtones) acceptèrent le plus souvent la notion que les Maori « devenaient des Britanniques à la peau brune [Brown Britons] par mariage, par l'individualisation, par la modernisation et l'assimilation ${ }^{33}$ ». En 1920, le député maori Apirana Ngata demanda au Parlement si la loi qui restreignait le retour en NouvelleZélande de résidants néo-zélandais non-blancs ayant quitté le pays s'appliquait aux Maori. Le premier ministre conservateur William Massey lui répondit : «Dans ce cadre, le Maori est considéré comme un Européen. Les Maori sont de très bons citoyens, ils ont les mêmes droits et les mêmes privilèges que les Européens, et ils les méritent $t^{34}$ ». Dans les deux sens du terme, les Maori étaient considérés comme assimilables aux Blancs.

11 En Australie, il ne fut jamais question d'accorder aux Aborigènes une place d'égaux dans la société blanche. Les politiques « assimilationnistes » tardivement déployées à l'égard d'enfants métis, et appliquées par la force, ne visèrent nullement à assimiler tous les Aborigènes. La ségrégation s'appliqua sans discontinuité aux full-bloods - les autochtones au «sang non-mêlé ». Elle se "justifiait » au moyen de trois convictions. D'une part, les Aborigènes étaient trop "primitifs "pour pouvoir être «civilisés». En second lieu, l'Australie blanche ne pouvait tolérer la présence de citoyens noirs, égaux en son sein. Antithèse des Maori, les Aborigènes avaient la peau très sombre, des traits physiques différents de ceux des Blancs, un niveau d'« avancement » technologique perçu comme le plus faible du monde, et ils ignoraient l'agriculture. Ils furent placés « au dernier rang, ou presque, d'une échelle hiérarchique humaine ${ }^{35}$ ». Si les Maori étaient les Noirs les plus «civilisés » du monde britannique, les Aborigènes furent situés à leur opposé. Enfin, la certitude que les Aborigènes allaient disparaitre d'eux-mêmes rendait futile toute notion d'assimilation. Le "problème aborigène " se résoudrait en isolant les survivants jusqu'à ce que leur « race » expire.

La fierté « raciale » et le désir d'homogénéité - source de certitude identitaire - ne furent bien entendu pas les seuls fondements des affirmations identitaires nationales australiennes et néo-zélandaises à la fin du XIX ${ }^{e}$ siècle et au cours des deux premiers tiers $\mathrm{du} \mathrm{XX}^{\mathrm{e}}$. Mais leur importance - aux côtes de valeurs censément nationales telles la débrouillardise coloniale ou la solidarité entre mates $^{36}$ - fut néanmoins primordiale. Le 
démantèlement des politiques discriminatoires fut toutefois une initiative politique bipartite.

13 Les Aborigènes australiens obtinrent le droit de vote au niveau fédéral en $1962^{37}$. En 1967 le gouvernement du conservateur Harold Holt abolit, par référendum et avec le soutien des Travaillistes, les clauses discriminatoires de la Constitution à l'égard des Aborigènes. En 1972, le travailliste Gough Whitlam fut élu en promettant de mettre fin à toute politique raciste, que ce fût à l'encontre des Asiatiques ou des Aborigènes ${ }^{38}$. Il déclara que le gouvernement ne rechercherait plus l'assimilation des Aborigènes; que ces derniers deviendraient maîtres de leur destin, avec l'assistance du gouvernement pour surmonter les difficultés auxquelles ils faisaient face ; qu'ils seraient libres de maintenir leur identité s'ils le souhaitaient. Il perdit le pouvoir en 1975, mais ses successeurs, des deux bords, poursuivirent sa politique. Quant à la politique de l'Australie blanche, elle fut définitivement enterrée par Gough Whitlam en 1975, avec notamment la loi Racial Discrimination Act, rendant illégale toute discrimination fondée sur la «race». La Nouvelle-Zélande avait adopté une loi similaire en 1971.

14 Cette inversion de l'un des aspects centraux de la politique australienne depuis l'unification ne fut pas sans conséquences. «La restructuration des lois sur l'immigration au début des années 1970 représenta une césure radicale dans le discours national officiel, non seulement au sujet de qui pouvait, maintenant, être formellement inclus dans «le peuple australien ", mais aussi au sujet de l'image préférée qu'aurait la nation d'ellemême ${ }^{39}$. » Brièvement, Canberra et Wellington prônèrent l'assimilation de ces nouveaux immigrés, qui devraient renoncer à leur culture d'origine. En Australie, toutefois, le terme de «multiculturalisme»- emprunté au Canada - fit sa première apparition majeure en politique dès 1973, lorsque le Ministre de l'Immigration Al Grassby publia un article intitulé «Une Société multiculturelle pour l'avenir ${ }^{40}$ ». Le multiculturalisme en tant que doctrine d'État en Australie émergea réellement sous le gouvernement travailliste de Bob Hawke dans les années 1980. Le gouvernement encouragea explicitement les minorités, aborigènes ou d'origine immigrée, à conserver et à pratiquer leur culture d'origine. En 1989, le gouvernement publia un Programme national pour une Australie multiculturelle (National Agenda for a Multicultural Australia), qui reçut le soutien de l'opposition conservatrice. Aujourd'hui, les autorités australiennes placent le multiculturalisme au cœur de l'identité de la nation, tandis qu'en Nouvelle-Zélande, depuis les années 1980, le gouvernement promeut officiellement un "biculturalisme » (union des Maori et des Pakeha, Néo-Zélandais non-autochtones), et de facto une diversité culturelle bien plus large ${ }^{41}$.

La Nouvelle-Zélande et l'Australie ont ainsi connu un parcours similaire, en matière de discours et de politique identitaires, affirmant tout d'abord une nécessaire homogénéité fondée sur la certitude d'une identité raciale "objective", avant de tolérer puis, très rapidement, de promouvoir, la diversité ethnique et culturelle. Les autres pays de la région, sous administration impériale jusque dans les années 1960 ou 1970, et demeurés à deux exceptions près - majoritairement autochtones, ont fait l'expérience d'un parcours différent, bien que similaire par certains points. 


\section{Configurations identitaires dans les îles du Pacifique}

\section{Politiques coloniales}

Les Fidji britanniques, sous l'administration d'Arthur Gordon et de ses successeurs, initièrent une politique de « préservation » des indigènes, qui allait influencer les autres colonies de la région. A. Gordon, confronté dès son arrivée en 1875 à une épidémie de rougeole dévastatrice, déclara que la "continuation de l'existence de la race fidjienne dépend de la préservation de ses traditions, contre les influences corruptrices de la communauté des planteurs ${ }^{42}$ ». Philip Mitchell, Gouverneur des Fidji de 1942 à 1945, affirma notamment qu' " il est généralement reconnu que la communauté villageoise forme la base de la société fidjienne et qu'il serait idiot, et même pernicieux, de la part du gouvernement d'intervenir pour la détruire ${ }^{43}$ ”. Extraire les Fidjiens de leur identité collective traditionnelle et les reconstituer en individus « libres »et autonomes, dénués de leurs réseaux d'entraide coutumiers, aurait des effets destructeurs et serait injustifiable. Cette approche protectrice s'effectua par le biais de politiques discriminatoires et ségrégationnistes. L'alcool ayant fait des ravages dans le Pacifique, les Fidjiens, même les chefs de plus haut rang, ne purent s'en procurer qu'au moyen d'un permis, règle qui ne s'appliquait pas aux Blancs ${ }^{44}$. Plus généralement, le gouvernement limita autant que possible les rapports entre colons blancs et Fidjiens, et "[l] eur isolement de la société coloniale dominante était quasi absolu»" des Fidjiens furent confinés en milieu rural, maintenus à l'écart du développement de la colonie, jusque dans les années 1960. En 1946, le député blanc A.A. Ragg affirma : « [L] e temps est venu [...] de souligner les termes de l'Acte de Cession assurant la sauvegarde des intérêts de la race fidjienne, et d'offrir la garantie que Fidji sera préservé et maintenu en tant que pays pour les Fidjiens, à tout jamais ${ }^{46} »$. Les Îles Fidji, à l'inverse de l'Australie, n'avaient pas pour vocation de devenir un "pays de l'homme blanc »- encore moins un pays où une importante population indienne immigrée serait dotée d'une réelle influence politique. Sir Arthur Grimble, qui gouverna les Kiribati et les Tuvalu de 1926 à 1932, insista lui aussi sur la préservation des coutumes autochtones ${ }^{47}$, et adopta une politique similaire. Sir Hubert Murray, Gouverneur de la Papouasie australienne de 1908 à 1940, s'inspira de la politique « protectrice » d'Arthur Gordon aux Fidji, et, en 1925, un décret ( Uncontrolled Areas Ordinance) interdit aux Blancs de quitter les territoires directement sous le contrôle du gouvernement pour s'aventurer en terres papoues, le but avoué étant de protéger les Papous face aux incursions blanches ${ }^{48}$. Les autochtones furent confinés à leurs villages.

17 Pour autant, l'une des conséquences de la colonisation et de ses politiques fut le développement de sociétés pluriethniques, rassemblant non seulement des Blancs et des autochtones aux origines diverses, mais également des communautés asiatiques plus ou moins importantes - travailleurs sur les plantations ou commerçants indépendants. En 1908, le Colonial Office à Londres demanda aux autorités de tous les territoires et protectorats britanniques dans le Pacifique de ne pas autoriser le recrutement collectif de travailleurs chinois. Les immigrés chinois individuels et indépendants pouvaient être tolérés, si les autorités locales le souhaitaient. En 1918 le gouvernement fidjien s'opposa donc à une requête de la Planters Association, qui souhaitait recruter des Chinois en nombre conséquent suite à la cessation de l'« importation» de travailleurs indiens ${ }^{49}$. Malgré tout, les Chinois des Fidji, ainsi que des autres territoires directement sous 
administration britannique, échappèrent à la discrimination bien plus flagrante imposée aux Chinois des territoires australiens et néo-zélandais. Aux Îles Salomon, dirigées par les Britanniques, les quelques Chinois - principalement des commerçants indépendants n'attirèrent pas l'animosité du gouvernement ${ }^{50}$. De même, au Vanuatu, condominium franco-britannique, il y eut peu de discrimination institutionnelle à leur encontre. Malgré les plaintes de boutiquiers français et britanniques face à la concurrence, le commerce chinois ne fut pas entravé ${ }^{51}$.

18 À Nauru, à l'inverse, île à phosphate sous administration australienne, le gouvernement colonial mit en place une "politique d'apartheid $»^{52}$, interdisant aux Chinois de commercer avec les autochtones, et d'entrer dans leurs maisons pour quelque raison que ce fût. Un « quartier chinois » fut établi sur l'île, et soumis à un couvre-feu permanent; pour la "protection" des autochtones, il ne devait y avoir aucune rencontre entre Chinois et Nauruans après la tombée de la nuit. Et ce d'autant que les Chinois de Nauru furent proportionnellement bien plus importants que dans tout autre territoire du Pacifique. Ils étaient plus de 1500 à la fin des années $1920^{53}$, dépassant légèrement la population autochtone, qui franchit la barre des 1500 personnes en 1932. En Papouasie australienne, l'immigration chinoise fut tout simplement interdite ${ }^{54}$.

Lorsqu'ils prirent le contrôle des Samoa, les Néo-Zélandais expulsèrent plus de 1300 des 2200 membres de la communauté chinoise en l'espace de deux ans - avant d'autoriser une nouvelle vague migratoire d'environ 3000 personnes dotées des contrats de travail dans les années 1920, puis de procéder à nouveau à l'expulsion de Chinois, pourtant résidents de longue date, en 1938 et après la Seconde Guerre mondiale. Les autorités néozélandaises interdirent à ces immigrés d'épouser des Samoanes, et « le concubinage [fut] combattu avec force $»^{55}$. En 1923, le Gouverneur George Richardson demandait ainsi aux autorités d'« empêcher la souillure jaune [...] de polluer la race britannique et les autres races $»^{56}$, samoane et îliennes. Cette mesure eut pour double effet de restreindre les rapports entre les communautés, et de freiner l'implantation durable et l'accroissement démographique de la population chinoise, essentiellement masculine. Plus généralement, la discrimination infligée aux Chinois dans les territoires australiens et néo-zélandais refléta les politiques «raciales »adoptées dans les métropoles de ces deux micropuissances.

Alors que les Chinois s'installèrent dans l'ensemble du Pacifique, sans jamais constituer une proportion importante de la population de leur terre d'« accueil »- sauf à Nauru -, les Indiens n'immigrèrent qu'aux Fidji, où ils formaient, à la veille de l'indépendance en 1970, environ 50 \% de la population ${ }^{57}$. De 1879 à 1916, la Colonial Sugar Refining Company fit venir près de 63000 Indiens, venus travailler sur les plantations de la compagnie pour une durée de cinq ans, renouvelable. Autorisés à immigrer avec leur famille, $60 \%$ restèrent aux Fidji à l'expiration de leur contrat ${ }^{58}$. Leur présence suscita peu à peu l'inquiétude. Dès 1924, un homme politique blanc remarquait que, «[a] u vu du grand nombre de travailleurs indiens à Fidji, le maintien d'une majorité fidjienne adéquate a suscité une profonde réflexion ${ }^{59}$ ». Le " problème indien » était posé, articulé autour de l'idée que les intérêts des autochtones pâtiraient de leur mise en minorité. En 1936, il y avait 85000 Indiens aux Fidji, représentant près de $43 \%$ de la population de l'archipel. Environ 60000 d'entre eux étaient nés aux Fidji. Il s'agissait dès lors d'une communauté propre à la colonie, non plus simplement indienne mais « indo-fidjienne ».

21 Le recensement de 1946 marqua une année charnière, puisque, pour la première fois, il y avait plus d'«Indiens» $(46,4 \%)$ que de Fidjiens autochtones $(45,4 \%)^{60}$. En 1956, ces 
chiffres étaient de $49 \%$ et de $42,8 \%$; l'écart s'accentuait. Dans ce contexte, les autorités coloniales et les chefs autochtones s'inquiétèrent des revendications de porte-parole de la communauté indienne, qui réclamaient l'égalité en droits de tous les sujets britanniques dans la colonie, ainsi que l'instauration d'un suffrage universel sans distinction de "race ». Les autorités répondirent à ces revendications en "se référant à leur devoir concernant la protection des intérêts des Fidjiens autochtones ». Ils soulignèrent "le caractère 'primitif'des Fidjiens, et donc leur vulnérabilitét ${ }^{61}$ ». En 1946, notamment, A. A. Ragg, membre «européen» du Conseil législatif, passa sommairement en revue la place des trois principales «races» dans la colonie. Les Fidji appartenaient aux Fidjiens, affirma-t-il, mais la présence des Blancs était légitimée par leur fonction protectrice envers les autochtones, en accord avec l'Acte de Cession. Les Indiens, pour leur part, n'étaient que des étrangers ${ }^{62}$. Cette logique devait conforter le soutien des autochtones au gouvernement colonial, en présentant comme nécessaire une résistance commune aux demandes indiennes. Ses répercussions marquèrent profondément la vie politique fidjienne post-coloniale, et ce jusqu'à ce jour. En misant sur une forme de partenariat stratégique entre chefs fidjiens et Blancs, le gouvernement colonial rendait par avance très problématique la constitution d'une identité nationale post-coloniale, commune à tous les citoyens de l'archipel.

\section{Politiques identitaires et nationalité au moment de l'indépendance}

Les Constitutions proclamées par les États nouvellement indépendants condamnent et interdisent la discrimination « raciale». Sur presque tous les plans, les résidents d'origine étrangère bénéficient des mêmes droits que les autochtones ; ceci est vrai également pour ce qui est de la citoyenneté. Tous les pays admettent la naturalisation des étrangers, par naissance, par mariage ou par immigration, sujette à diverses restrictions et obligations. La nationalité n'est donc pas « ethnique ». Nauru, néanmoins, constitua longtemps une exception. La Constitution conféra la citoyenneté nauruane aux personnes définies comme étant de « race » nauruane par un décret colonial de 1956, et à leurs descendants, tout en stipulant que le Parlement pouvait légiférer pour étendre ces dispositions (art. 75.1). Seules les femmes épousant un Nauruan, et les apatrides nés à Nauru, pouvaient être naturalisés. Puisque le Parlement ne légiféra pas en la matière avant trente-sept ans, les résidents blancs, chinois et autres non-indigènes, majoritaires à la veille de l'indépendance ${ }^{63}$, étaient privés de la citoyenneté nauruane. Étant l'un des deux seuls pays îliens - avec les Fidji- confrontés à une population non-autochtone importante, Nauru opta ainsi de privilégier ses indigènes. Par voie de conséquence, seuls les autochtones (ou presque) pouvaient voter, être élus, et diriger le pays. L'article 75.1 ne donna lieu à une nouvelle législation qu'en 2005, lorsque le Parlement adopta la loi Naoero Citizenship Act, autorisant les étrangers à solliciter leur naturalisation s'ils étaient mariés à un (e) Nauruan (e) depuis au moins dix ans, dont trois au moins passés sur l'île. Leur requête devait être examinée par le Conseil des ministres (art.5). En 2006, la population non-autochtone de Nauru était estimée à 464 personnes (5\%), dont 93 «Européens » et 273 "Chinois $»^{64}$. Ces derniers, résidents permanents, descendants de colons chinois, souvent gérant des boutiques et des restaurants sur l'île ${ }^{65}$, étaient donc - pour la plupart privés de la citoyenneté de leur pays de résidence.

Sur le plan législatif, les Samoa établirent un Parlement monocaméral composé de quarante-neuf députés, sélectionnés selon des critères ethniques. Les citoyens non- 
autochtones - principalement blancs ou d'origine chinoise - élisaient deux représentants, tandis que les autres sièges étaient réservés à des candidats élus (au suffrage universel depuis $1990^{66}$ ) parmi les matai, les chefs de communautés familiales indigènes ${ }^{67}$. En 2009, la loi Electoral Amendment Act étendit l'obligation d'être matai aux candidats pour les deux sièges réservés aux élus des minorités ethniques. Début 2011, briguant avec succès un nouveau mandat, le premier ministre Sailele Malielegaoi déclara que cette décision était sa plus grande source de fierté. Elle avait établi une "égalité " au sein de l'assemblée législative, dit-il, et «[c]'est la première fois [...] que notre parlement est pleinement samoan $^{68}$ ». En pratique, cela signifie que les candidats aux sièges des députés nonautochtones doivent obtenir un titre de matai, et donc appartenir - si ce n'est que par le biais de l'adoption - à une famille autochtone. Aux élections législatives de 2011, les candidats Pat Ah Him et Niko Lee Hang, ayant chacun obtenu un titre de matai, remportèrent ces sièges ${ }^{69}$. La mesure ne constituait donc pas une exclusion totale des " afakasi » ou des citoyens non-indigènes, mais symbolisait néanmoins une « autochtonisation » accrue des instances dirigeantes.

Fidji fut le seul autre pays à subdiviser les citoyens en listes électorales ethniques. En vertu de la seconde section du chapitre 5 de la Constitution de 1970, tous les électeurs seraient inscrits sur une liste nationale, mais également sur l'une des trois listes « raciales»: celle des «Fidjiens» (autochtones), celle des «Indiens», et celle des personnes d'une autre appartenance ethnique. Par le biais d'un système complexe, chaque personne pouvait placer quatre bulletins dans l'urne : deux pour élire des députés de sa propre "race", et un pour un député de chacune des deux autres catégories ethniques. La Constitution définit juridiquement les appartenances « raciales " pour la composition de ces listes. Était «fidjienne » (Fijian) toute personne dont le père, ou l'un des ancêtres dans la lignée paternelle, était le fils de deux parents autochtones fidjiens, ou de deux parents autochtones de n'importe quelle île du Pacifique, ou d'un parent de chacune de ces deux catégories. Seule une personne correspondant à ces critères pouvait s'inscrire sur la liste électorale « fidjienne ».

La «fidjianité » était donc « biologique », la Constitution ne prévoyant aucune possibilité pour une personne de redéfinir ou de contester son appartenance 'raciale'; de plus, tout électeur devait être catégorisé. En outre, le choix du terme est significatif. Si une personne non-« fidjienne » pouvait être citoyenne, elle ne pouvait se considérer fidjienne, bien qu'elle fût citoyenne des Fidji. «Race» et nationalité se voyaient sémantiquement brouillées; une personne de "race » indienne ou blanche peinerait à qualifier sa propre nationalité. Cette définition n'encourageait pas les "Indiens " à s'identifier à la nation fidjienne. Ceci était d'autant plus vrai qu'un immigré polynésien de première génération devenait « fidjien », tandis que les descendants d'Indiens établis aux Fidji depuis plusieurs générations ne l'étaient pas.

Ces dispositions extraordinairement compliquées garantissaient qu'il y aurait toujours vingt-deux autochtones à la Chambre des Représentants, soit autant que d'« Indiens ", malgré le fait que la population indo-fidjienne était plus importante que celle des indigènes. Cette "parité » contrebalançait un léger déséquilibre démographique, et se prémunissait par avance des effets de la croissance démographique «indienne »: les « Fidjiens » ne seraient pas mis en minorité au Parlement par les effets de la démocratie.

La facilitation d'une identité nationale ancrée dans les esprits avait une valeur pratique impérative aux yeux des gouvernements, au moment de l'indépendance. L'autorité de l'État sur l'ensemble du territoire, condition nécessaire à la garantie de l'ordre et de la 
stabilité, dépendait de l'adéquation entre «État » et «nation ». Si la nation ne constituait pas une réalité identitaire ressentie par les citoyens, la légitimité de l'État devenait problématique, tout particulièrement dans les régions reculées. Dans ce domaine, les mesures de promotion identitaire aux Fidji ont été fluctuantes, voire contradictoires. Le gouvernement de Ratu Kamisese Mara vit dans les cérémonies d'indépendance l'occasion de mettre en scène, pour la première fois, une image de l'identité du pays. Bien qu'il ait été élu en s'appuyant principalement sur l'électorat autochtone, il prit soin de souligner le caractère multiethnique et multiculturel du pays. Les festivités célébrant l'indépendance durèrent une semaine à Suva, et plusieurs jours dans le reste du pays. Elles incorporèrent des rituels et des représentations culturelles de toutes les communautés ethniques, accordant la primauté - à égale mesure - aux aspects autochtones et indiens. Les autorités présentèrent le pays comme un "tabouret à trois pieds ", reposant sur les apports des indigènes, des «Indiens » et des "Européens », les autres minorités étant associées à ces derniers. La métaphore du tabouret était certes ethnicisante, reléguant les citoyens individuels à leurs «races » distinctes, mais elle construisait l'image d'une unité nationale respectueuse des différences, où chaque communauté était essentielle à la nation: Sans les «Indiens ", les Fidji ne seraient plus qu'un «tabouret à deux pieds »... Le festival hindou de Diwali et l'anniversaire du prophète Mahomet furent reconnus comme jours fériés; le christianisme autochtone n'occulterait pas les religions indiennes. Au cours des années 1970, toutefois, le Parti de l'Alliance reposa de plus en plus nettement sur l'électorat autochtone, et les cérémonies nationales - lors de l'accueil de dignitaires étrangers, par exemple - accentuèrent la place des rituels autochtones, réduisant puis éclipsant les représentations de la culture indienne. Sous le gouvernement de l'Alliance, la nation en venait à être assimilée à son ethnie indigène, bien que les minorités ne fussent jamais totalement exclues ${ }^{70}$.

La démocratie fidjienne a été confrontée aux écueils d'une vie politique - et plus largement d'une société - cadrées par des identifications « raciales ». En 1995, Jai Ram Reddy, chef officiel de l'opposition, déclara : [N] ous ne sommes pas une nation. [...] [N] ous sommes des étrangers culturels [cultural strangers], nous ne connaissons pas nos voisins, nous ne connaissons pas leur langue, nous ne connaissons pas leur système de valeurs $»^{71}$. Il n'existait pas de "valeurs nationales », ciment nécessaire à toute nation, mais uniquement des valeurs communautaires. Si l'anglais servait de langue nationale, les « Indiens » méconnaissaient la langue fidjienne. Aux yeux de Ratu Joni Madraiwiwi ${ }^{72}$, les premiers ministres autochtones du pays - Kamisese Mara (1970-87, 1987-92), Sitiveni Rabuka (1992- 99) et Laisenia Qarase (2000-06) - ont tous encouragé une unité ethnique indigène plutôt qu'une unité nationale pluri-ethnique; Timoci Bavadra, brièvement premier ministre en 1987, étant la seule exception. J. Madraiwiwi ajoute : «[L] a plupart [des personnalités politiques fidjiennes] ont de réelles difficultés à concevoir collectivement toutes les communautés ethniques sous le terme de 'nous'. Leur discours "nous" oppose toujours à 'eux', tout particulièrement lorsqu'il s'agit des Indo-Fidjiens " "73. Fidji ne possède pas d'identité nationale, affirme-t-il. Le pays est multiculturel, mais ses différentes communautés ne se reconnaissent pas une identité commune. Vijay Naidu, Professeur à l'Université du Pacifique Sud, évoque pour sa part un "État fidjien ethnicisé [incapable] de créer une "nation" à partir des citoyens culturellement divers des Fidji $»^{74}$. Cette incapacité relèverait en grande partie d'un manque de volonté, dû à des intérêts politiques profondément ancrés. Les conservateurs autochtones, au pouvoir quasiment sans interruption depuis l'indépendance, s'appuient sur l'électorat indigène, mobilisé en tant que tel. Le Département de la Culture du gouvernement insiste aujourd'hui sur l'unité nationale - mais se réfère à la diversité 
culturelle de la population, plutôt qu'à une culture nationale, pluri-ethnique mais partagée, émergeant de cette diversité ${ }^{75}$. Son multiculturalisme intègre les minorités d'origine immigrée, mais peine à proposer une unité culturelle inclusive. La notion de vakavanua - la "voie de la terre»-, avancée par les dirigeants indigènes comme fondement de l'identité nationale, est spécifique aux autochtones. Les terres étant réservées aux "Fidjiens", les minorités ne peuvent se réclamer de cette culture «nationale » ou de ce mode de vie.

Par-delà le cas fidjien, toute invocation de la «tradition $»^{76}$ et de l'histoire risquait, et risque, de brouiller la distinction entre les citoyens d'un pays et sa population autochtone. Les implications pour les citoyens non-autochtones sont problématiques. Les références politiques aux Papou-Néo-Guinéens, aux Samoans ou autres, sont souvent ambiguës : désignent-elles une nationalité, une appartenance ethnique, ou confondentelles les deux? La rhétorique traditionaliste sous-entend que les immigrés et leurs descendants soient ou bien intégrés à une nation reposant sur des valeurs autochtones qui deviendraient les leurs, ou bien exclus de la conception de la nation. La «culture » promue par les autorités est celle des autochtones. Le Centre culturel du Vanuatu, par exemple, a pour fonction de rechercher, de promouvoir et de préserver les cultures indigènes de l'archipel, sous toutes leurs formes et dans leur pluralité ${ }^{77}$, sans s'intéresser aux particularités des minorités chinoise, française ou vietnamienne, par exemple.

Pour autant, les minorités d'origine immigrée ne sont pas toujours exclues d'un imaginaire national défini par les autorités. Aux Samoa par exemple, le gouvernement demande aux enseignants de sciences sociales au collège d'encourager les élèves à « voir le monde tel que d'autres personnes le voient", en leur permettant, entre autres, de comprendre les valeurs de personnes issues de ces minorités ${ }^{78}$. La nation et l'identité samoanes doivent ainsi s'extraire au moins partiellement, à travers l'éducation, d'un modèle purement autochtone. De manière plus ponctuelle, la médaille d'or obtenue par le nageur blanc papou-néo-guinéen Ryan Pini aux Jeux du Commonwealth en 2006 fut décrite par le premier ministre Michael Somare comme « faisant partie de la construction de la nation », un «événement [qui] toucha tous les Papou-Néo-Guinéens », un " puissant facteur d'unification $»^{79}$. Bien que non-autochtone, ce héros sportif portait haut les couleurs du pays, et contribuait - aux yeux des autorités - à souder la nation. Dans ce cadre, sa nationalité primait bien évidemment sur ses origines ou sa couleur de peau.

\section{La tentation du rejet}

31 Aujourd'hui, dans l'ensemble des pays océaniens issus de l'Empire britannique, la diversité ethnique et culturelle est admise, voire - notamment en Australie et en Nouvelle-Zélande - promue et célébrée. Malgré des restrictions durables dans certains pays (Nauru, Samoa, Fidji), sur le plan civique ou politique, les minorités d'origine immigrée sont intégrées à la société nationale, et leurs membres exercent parfois un rôle de premier plan dans le domaine politique, économique, culturel ou encore sportif. Néanmoins, la décennie 2000 a été marquée, dans toute la région, par des mouvements populaires d'hostilité à l'encontre de communautés d'origine asiatique; dans cinq pays (Australie, Fidji, Salomon, Tonga, Papouasie-Nouvelle-Guinée), ces mouvements ont donné lieu à des actes de violence; dans d'autres, à l'expression d'une sinophobie plus ou moins importante. Ainsi, alors même que leurs dirigeants prônent un rapprochement diplomatique et économique avec les puissances d'Asie, les Océaniens, aux portes de cette 
région, sont parfois tentés de bannir de la communauté nationale leurs concitoyens qui en sont originaires.

En Australie et en Nouvelle-Zélande, l'État se fit l'architecte de la (re)définition identitaire du pays, cherchant à altérer l'image que pouvaient avoir les citoyens de leur nation. Cette initiative de l'État faisait suite à l'altération, là aussi par l'État, des politiques d'immigration, et donc de la composition ethnoculturelle de la population. Il n'y eut pas de pression populaire significative en faveur de l'abolition de l'Australie blanche; les gouvernements australiens des deux bords - dans les années 1970 et 1980 - promurent activement le multiculturalisme, face à l'opposition grandissante d'une partie de la population. Dans ce contexte, certains Blancs aujourd'hui, refusant de se subsumer dans une « culture » pluri-ethnique, expriment une hostilité marquée envers leurs concitoyens d'origine non-européenne. En 1984, l'historien australien Geoffrey Blainey argua que le taux d'immigration asiatique dépassait le seuil de tolérance de la population d'accueil, et qu'il devait être réduit pour maintenir la cohésion et la paix sociale ${ }^{80}$. Cette opinion généra un début de controverse publique, reprise et amplifiée par la suite. Selon G. Blainey, les Australiens différencieraient toujours leurs concitoyens selon leur couleur de peau - et, implicitement, se méfieraient des Australiens d'apparence asiatique, s'ils étaient visibles en grand nombre. Accusé d'être lui-même hostile à un accroissement de la minorité 'asiatique', l'historien, attaché à la notion d'héritage britannique de la nation, ne démentit pas. L'idée selon laquelle la population blanche devait demeurer largement majoritaire était désormais posée. John Howard, chef de l'Opposition, allait bientôt reprendre ce point de vue à son compte ${ }^{81}$.

En août 2001, un navire norvégien, le Tampa, recueillit en haute mer 433 Afghans entassés dans un bateau de pêche, et se dirigea vers les côtes australiennes. John Howard, devenu Premier ministre, interdit au Tampa de pénétrer les eaux territoriales de l'Australie. Lorsque le navire entra malgré tout dans les eaux australiennes, il fut intercepté, et les Afghans expédiés dans un centre de détention à Nauru, avec la permission des autorités nauruanes. John Howard, alors en campagne électorale, afficha sa décision comme le symbole de sa politique, la présentant comme une défense des intérêts australiens face à des immigrés indésirables ${ }^{82}$. Dans le contexte qui suivit les attentats de New York en septembre, le gouvernement Howard fut soutenu par l'opinion publique. $77 \%$ des Australiens se dirent d'accord avec ses actes lors de l'affaire du Tampa, et il fut réélu en novembre avec $51 \%$ des voix; les sondages avant le mois d'août avaient été nettement favorables à ses adversaires travaillistes ${ }^{83}$.

En 1996, le parti New Zealand First intégrait un gouvernement de coalition conservateur. Son fondateur Winston Peters - de père pakeha et de mère maori - bâtit sa rhétorique sur une réactualisation du " péril jaune "; il y avait, à ses yeux, trop d'Asiatiques en NouvelleZélande ${ }^{84}$. La même année, un sondage indiqua que $60 \%$ des Néo-Zélandais partageaient cette opinion, l'étendant aux immigrés originaires des îles du Pacifique ; $40 \%$, en outre, pensaient que les Asiatiques et les îliens apportaient une concurrence malvenue sur le marché de l'emploi, privant certains Néo-Zélandais « natifs » d'accès au travail ${ }^{85}$. En soi, le sondage normalisait d'ailleurs la question, indiquant aux tenants d'opinions racistes que leur question pouvait légitimement être posée, et les informant qu'ils s'inscrivaient dans une mouvance majoritaire. En 1996, également, Pauline Hanson fit son entrée au Parlement fédéral australien. Élue députée indépendante, elle fonda par la suite le parti One Nation, dont le thème d'unité nationale reposait sur l'exclusion des personnes nonblanches. Son premier discours au Parlement fut fortement médiatisé. Se présentant 
comme une femme ordinaire, elle affirma être la voix du bon sens en dénonçant les aides dont bénéficiaient les Aborigènes, et ajouta :

Comme la plupart des Australiens, je veux une révision radicale de notre politique d'immigration, et l'abolition de celle du multiculturalisme. Je crois que nous sommes en danger d'être submergés par des Asiatiques. Entre 1984 et 1995, 40 \% de tous les migrants entrant dans ce pays étaient d'origine asiatique. Ils ont leur propre culture, leur propre religion, ils forment des ghettos ${ }^{86}$ et ne s'assimilent pas. [...] Un pays vraiment multiculturel ne peut jamais être fort ou uni.

Confondant "multi-ethnique » et "multi-culturel», elle déclara ne vouloir aucune personne non-blanche supplémentaire dans le pays. Ses paroles ravivèrent la rhétorique du «péril jaune » :

Le temps nous manque. Il ne nous reste peut-être que 10 à 15 ans pour inverser la tendance. En raison de nos ressources et de notre position dans le monde, nous n'aurons pas notre mot à dire car nos pays voisins comme le Japon, avec 125 millions de personnes; la Chine, avec 1,2 milliard de personnes ; l'Inde, avec 846 millions de personnes; l'Indonésie, avec 178 millions de personnes; et la Malaisie, avec 20 millions de personnes sont tout à fait conscients de nos ressources et de notre potentiel. Éveille-toi, Australie, avant qu'il ne soit trop tard.$^{87}$

Cette hantise ancestrale, rénovation des peurs du XIX ${ }^{e}$ siècle, trouva un écho favorable au sein d'une frange de l'électorat. Le phénomène One Nation fut, en soi, de courte durée. En juin 1998, le parti remporta onze sièges sur 89 au Parlement du Queensland, mais aucun à la Chambre des Représentants fédérale - et un seul au Sénat fédéral - en octobre de la même année. Il avait obtenu près de $8,5 \%$ des voix sur le plan national, faisant de lui le troisième parti derrière les Libéraux et les Travaillistes, mais n'était arrivé en tête dans aucune circonscription ${ }^{88}$. Pauline Hanson elle-même perdit son siège. En 2001, son parti disparut totalement du Parlement. Son influence dans le débat public, toutefois, fut durable. Quant à New Zealand First, il obtint dix-sept sièges (sur cent vingt) en 1996, cinq seulement en 1999, puis treize en 2002, sept en 2005, et aucun en 2008. Winston Peters, député depuis 1978 sous diverses étiquettes, perdit alors son siège pour la première fois ${ }^{89}$. Par le jeu des coalitions, il avait été vice-Premier ministre et Trésorier dans un gouvernement conservateur de 1996 à 1998, et Ministre des Affaires étrangères dans un gouvernement travailliste de 2005 à 2008. L'une des figures les plus marquantes de l'histoire néo-zélandaise contemporaine semble aujourd'hui écartée du pouvoir éclaboussée notamment par un scandale financier-, mais il fut longtemps l'écho et le promoteur d'opinions qui ne peuvent avoir entièrement disparu. Ainsi, des deux côtés de la Mer de Tasman, Winston Peters et Pauline Hanson se faisaient un temps écho, introduisant avec fracas le thème du "péril jaune » dans le discours public et politique des deux pays.

$\mathrm{Au} \mathrm{XXI}{ }^{\mathrm{e}}$ siècle, en Australie, cette hostilité s'est focalisée également sur les musulmans bien qu'ils n'y constituent que $1,5 \%$ de la population ${ }^{90}$. Elle s'étend par ailleurs aux Arabes de toutes confessions. En décembre 2005, les « émeutes de Cronulla " (Cronulla riots ) choquèrent le pays. L'agression de deux Blancs, attribuée à des hommes d'origine arabe, et faisant suite à des incidents similaires, provoqua un déferlement de haine et de violence. Plusieurs milliers de Blancs, principalement de jeunes hommes, s'assemblèrent, et agressèrent systématiquement toute personne arabe qu'ils rencontrèrent. Quelques heures plus tard, des Blancs étaient agressés en retour par de petits groupes d'Arabes. La violence se poursuivit le lendemain, malgré les efforts de la police; des affrontements eurent lieu dans plusieurs banlieues de Sydney. Le rassemblement d'émeutiers blancs avait été coordonné spontanément; aucune organisation ne semblait l'avoir initiée ${ }^{91}$. En 
2006, la députée Danna Vale, ancienne Ministre des Anciens Combattants sous John Howard, appela les Australiennes à cesser d'avoir recours aux avortements, et à avoir plus d'enfants, car - dit-elle - les musulmanes en Australie étaient plus fécondes que les autres Australiennes $^{92}$. Le "péril jaune" justifiant autrefois les appels à une procréation patriotique s'était mué en 'péril islamique', lui aussi présenté comme démographique. La même année, Pauline Hanson, après plusieurs années de silence, prit la parole à nouveau pour dénoncer la «menace » que posaient les immigrés musulmans envers les traditions australiennes ${ }^{93}$.

En 2007, pour la première fois, dix-huit jeunes Australiens arabes, musulmans, devinrent des sauveteurs de bord de mer. Leur démarche était une réponse aux émeutes de Cronulla ${ }^{94}$. Les lifesavers se classent au sommet des « héros ordinaires » vénérés par de nombreux Australiens, et sont presque tous blancs. Il s'agissait ainsi, pour ces jeunes hommes, de proclamer, avec un symbolisme retentissant, leur appartenance à l'Australie, leur intégration, leur identité, la légitimité de leur présence. Il était possible d'être un musulman et un héros australien. En 2008, toutefois, les médias relayèrent la colère d'habitants de Camden, ville de la banlieue de Sydney, qui s'opposaient à la construction d'une école musulmane: "Ils sont en train de détruire l'Australie. Ils prennent le dessus. [...] Nous sommes tout à fait prêts à intégrer des Italiens, des Grecs, des Anglais, des Écossais - cette ville a toutes les nationalités. Les musulmans n'ont pas leur place dans cette ville - on est des Aussies, ok? $\|^{95}$. Les étrangers blancs pouvaient donc être " australianisés », mais il n'était pas concevable d'être australien et musulman. $\mathrm{Ni}$, d'ailleurs, blanc et musulman, l'islam étant assimilé à une "race " étrangère. Les lifesavers arabes n'étaient toujours pas australiens; ils ne pourraient jamais l'être. Peu après, un sondage suggéra qu'un Australien sur dix se déclarait ouvertement raciste, et que plus de $40 \%$ des habitants de cinq des six États croyaient que certains groupes ethniques ou culturels n'avaient pas leur place en Australie - le plus souvent, les musulmans ${ }^{96}$. De leur point de vue, la possibilité de se considérer australien n'était toujours pas ouverte à tous, et ne devait jamais l'être.

Dans bon nombre d'esprits, donc, le ressenti national peine à se dégager d'une adéquation à un sentiment d'appartenance "raciale " à visée exclusive. Il en est parfois de même dans les îles du Pacifique. La plupart de ces pays sont caractérisés par une grande homogénéité ethnique, la quasi-totalité de la population étant autochtone. Aux Tonga, $98,2 \%$ de la population est composée d'indigènes, ou de métis ayant un parent indigène. Il y avait, au recensement de 2006, 569 «Européens », 526 personnes originaires d'îles du Pacifique - dont plus de la moitié des Fidji -, et 646 « Asiatiques », dont 395 personnes s'identifiant comme étant d'appartenance ethnique chinoise ${ }^{97}$. La proportion d'autochtones - métis inclus - aux Samoa est estimée à 99,6\% $\%^{98}$ aux côtés de quelques Blancs et d'une infime minorité d'Asiatiques. Aux Kiribati, la population est autochtone à $99,4 \%$, métis compris ${ }^{99}$; le pays compte quelques centaines de personnes d'origine polynésienne, européenne ou chinoise. Tuvalu ne collecte pas de statistiques ethniques, mais sa population autochtone polynésienne est estimée à $96 \%{ }^{100}$, la quasi-totalité des autres personnes de nationalité tuvaluane étant les descendants d'immigrés micronésiens établis depuis des siècles sur l'atoll de Nui.

Les tensions interethniques y ont été quasi inexistantes. Les citoyens d'origine immigrée sont le plus souvent "autochtonisés" aux yeux de leurs concitoyens. La notion de fáaSamoa (mode de vie samoan), par exemple, est suffisamment intégrante pour permettre à un citoyen samoan de père indo-fidjien et de mère sino-samoane de parler de «notre fa'aSamoa »101. À la tête du Parti des Samoa (Samoa Party), Su'a Rimoni Ah Chong, 
d'origine chinoise, peut ainsi se présenter comme le défenseur de la propriété foncière collective autochtone, et dénoncer le caractère "non- samoan » (not Samoan) de projets de réforme du gouvernement ${ }^{102}$. L'actuel Procureur général du pays, Aumua Ming Leung Wai ${ }^{103}$, est également d'origine chinoise. Lorsqu'un député en 2005 suggéra que trop d'immigrés chinois s'installaient dans le pays, le Premier ministre Sailele Malielegaoi condamna fermement cette remarque, et qualifia son auteur de « raciste $»^{104}$. Malgré l'adéquation populaire entre culture autochtone et identité nationale, les Samoans se montrent, dans l'ensemble, ouverts à une certaine diversité ethnique - d'autant que les citoyens issus de minorités mettent en avant leur intégration et leur identité samoane.

41 Aux Kiribati, des ressentiments se sont exprimés à l'encontre de personnes d'origine chinoise, accusées de "prendre " les emplois des autochtones ${ }^{105}$. En juin 2009, une commission parlementaire fut chargée de déterminer si des commerçants immigrés avaient corrompu des hommes politiques pour obtenir leur permis de séjour. La commission devait se "concentrer sur la communauté des hommes d'affaires chinois, qui croît rapidement ${ }^{106}$. L'accroissement de la « communauté » chinoise et ses répercussions socioéconomiques généraient une certaine inquiétude. Néanmoins, Anote Tong, de père immigré chinois, fut élu Président de la République par ses concitoyens en 2003 - face à son frère Harry, candidat de l'opposition, et loin devant le seul candidat de parenté exclusivement autochtone, Banuera Berina. Il fut réélu en $2007^{107}$. Il demeure donc tout à fait concevable pour un citoyen d'origine immigrée d'être considéré pleinement iKiribati, au point d'aspirer aux plus hautes fonctions.

En Polynésie, ce n'est qu'aux Tonga qu'une sinophobie conséquente s'est exprimée. L'opposition à l'immigration chinoise a été articulée en lien étroit avec les revendications en faveur de la démocratie. En 1992, le journal du mouvement, le Ko e Kelea, titrait en première page : «Les Chinois et les Indiens vont prendre le contrôle : arrêtons-les maintenant, ou il sera trop tard ${ }^{108}$ ». Le ton rappelait la presse australienne du XIX ${ }^{\mathrm{e}}$ siècle, et anticipait les discours de Pauline Hanson à la fin du XXe. En 1998, le principal porte-parole du mouvement, "Akilisi Pohiva, déclara que le nombre de Chinois dans le pays générait des problèmes socio-économiques - privant d'emplois les autochtones -, et même "moraux " ${ }^{109}$. Cette hostilité s'est accrue au début du XXI ${ }^{e}$ siècle. Le journal Matangi Tonga, pourtant très critique à l'égard du mouvement démocrate, publie des lettres de lecteurs hostiles à la présence d'immigrés chinois ${ }^{110}$. En 2000, répondant aux requêtes de commerçants autochtones, un noble local à Nukunuku ordonna la fermeture de toutes les boutiques chinoises dans le district ${ }^{111}$. En 2001, une première vague d'agressions sinophobes marqua le pays; plusieurs boutiques furent incendiées, séparément. En novembre, le roi Taufa'ahau Tupou IV demanda à ses sujets de mieux accueillir les immigrés chinois - et de s'inspirer de leur éthique du travail. Quelques jours plus tard, toutefois, son fils cadet, le Premier ministre Prince 'Ulakalala Lavaka Ata, ordonna l'expulsion de six cent résidents chinois, cédant apparemment aux pressions populaires dans un contexte de chômage croissant ${ }^{112}$. Les partisans de cette décision arguèrent que les Chinois risquaient de « dominer » l'économie locale, à l'instar des «Indiens» aux Fidji ${ }^{113}$. Aux Tonga, bien plus que dans le reste de la Polynésie, apparaissent ainsi des échos du racisme australien de One Nation. La population dominante serait victimisée par une vague migratoire asiatique, inassimilable et décidée à exploiter la population locale. L'homogénéité et l'harmonie tongiennes en seraient menacées. Ce phénomène constitue une nouveauté dans le pays. Les démocrates ont ainsi accru leur popularité en attisant les craintes sinophobes d'une partie de la population, et en dénonçant le «laxisme» du gouvernement en matière 
d'immigration - jusqu'à ce que ce gouvernement ne les prenne de court en expulsant les victimes de ces discriminations et de ces violences.

En novembre 2006, les relations troubles entre le mouvement démocrate et les pulsions sinophobes d'une partie de la population - et de ses représentants - trouvèrent leur expression la plus violente. Une manifestation pour exiger l'instauration de la démocratie dégénéra en émeute, la première qu'ait connue le royaume depuis son unification au XIX ${ }^{\mathrm{e}}$ siècle. Le quartier chinois de Nuku'alofa fut incendié et ravagé ; six émeutiers présumés périrent dans l'un des incendies. "Presque toutes les boutiques chinoises du centre ville de Nuku'alofa » furent pillées et saccagées ${ }^{114}$, même si certaines furent protégées par leurs voisins autochtones ${ }^{115}$. Environ deux cents résidents de nationalité chinoise quittèrent le pays, avec l'assistance des autorités de Beijing ${ }^{116}$. Les attaques à l'encontre des Chinois furent condamnées par les Internautes tongiens sur les forums de discussion ${ }^{117}$. Si certains exprimèrent leur inquiétude ou leur hostilité face à des immigrés « païens » qui travaillaient le dimanche et concurrençaient les autochtones, la très grande majorité des participants soulignèrent les contributions positives des immigrés, et dénoncèrent les mauvais traitements à leur encontre. Même si cette tendance n'était peut-être pas représentative, elle suggère que le racisme sur lequel se sont appuyés certains hommes politiques est loin d'être généralisé.

La population des Fidji, quant à elle, compte 56,8 \% d'autochtones, 37,5 \% d'Indo-Fidjiens, et $5,7 \%$ de personnes d'autres appartenances ethniques ${ }^{118}$. Plus de 47000 citoyens des Fidji ne sont ni autochtones, ni « indiens ». Parmi eux, outre des îliens d'origines diverses, plusieurs milliers de Blancs et d'Asiatiques, presque tous d'origine chinoise. Ces communautés sont quasi invisibles, au sens où les «Indiens » sont la principale cible des ressentiments autochtones. Le rejet des « petites» minorités par les indigènes est presque inexistant - ou, du moins, peu étudié et peu médiatisé. Les gouvernements, même issus de coups d'État indophobes, ont été tolérants à l'égard des Sino-Fidjiens, recherchant - et obtenant - le soutien de personnalités politiques telles Paul Chan, Francis Hong Tiy, Yee Hon Wing ou James Ah Koy ${ }^{119}$. Quant aux citoyens blancs des Fidji, ils ne rencontrent presque aucune hostilité de la part de leurs compatriotes indigènes. Le stéréotype de l'Européen individualiste, matérialiste et mécréant, véhiculé ailleurs dans le Pacifique, est plutôt réservé aux 'Indiens'120. Dans les années 1990, en outre, le gouvernement nationaliste autochtone, héritier du coup d'État de 1987, reconduit au pouvoir par les élections de 1992 et de 1994, gouverna avec le soutien du Parti des Électeurs généraux ( General Voters'Party) ${ }^{121}$, représentant la majorité des électeurs blancs et asiatiques. Les porte-parole des «petites" minorités ont su s'associer aux autorités nationalistes indigènes, et leurs membres, éviter une xénophobie autochtone focalisée sur les « Indiens ».

$\mathrm{Au}$ recensement de 1999 - le plus récent -, le Vanuatu comptait 98,7\% d'autochtones ${ }^{122}$. La composition ethnique de la population non-indigène - soit 2349 personnes - n'était pas spécifiée, mais inclut des "Européens » et des «Asiatiques ", installés depuis l'ère coloniale, et leurs descendants. En 2006, l'ambassade de la République populaire de Chine au Vanuatu estimait que la population « chinoise » du pays était d'environ 600 personnes, dont la plupart étaient de petits commerçants urbains ${ }^{123}$. L'Association chinoise de Port Vila comptait, en 1998, soixante-cinq membres ${ }^{124}$. Soucieux de préserver une identité communautaire, ils n'attiraient pas d'hostilité notable de la part de leurs concitoyens. La Papouasie-Nouvelle-Guinée n'établit pas de statistiques ethniques, mais la population compte quelques milliers de personnes d'origine australienne, et d'immigrés chinois ${ }^{125}$, 
répartis dans l'ensemble des villes principales. Il n'y a pas, non plus, de statistiques officielles pour les Îles Salomon, mais la population autochtone mélanésienne est estimée à $93 \%$. Les descendants d'immigrés polynésiens installés depuis bien avant l'arrivée des Européens comprendraient $4 \%$ de la population, les $3 \%$ restants - soit 14000 personnes environ englobant des personnes d'origine immigrée plus récente, telles que les Blancs et les Asiatiques ${ }^{126}$.

En 2000, Paul Sillitoe affirmait qu'il y avait peu de «tensions raciales dans la Mélanésie contemporaine ", sauf aux Fidji, même s'il existait « une antipathie dans toute la région contre les personnes d'origine chinoise $»^{127}$. En 1997, les hommes d'affaires Moon Pin Kwan et Thomas Koh Chan, Salomonais d'origine chinoise, furent élus députés par les électeurs - presque tous autochtones - de Honiara et de Guadalcanal-ouest, respectivement ${ }^{128}$. Preuve que cette 'antipathie'était limitée dans la capitale et dans la province la plus 'cosmopolite'du pays ${ }^{129}$. De même, en 2001, l'historien John Waiko écrivait qu'il y avait " peu de racisme antiblanc » en Papouasie-Nouvelle-Guinée, et que " [1] es Chinois nés dans le pays sont, dans l'ensemble, acceptés comme étant des citoyens papou-néo-guinéens ». Il attribuait cette «absence de conflit ethnique » principalement au faible nombre de non-autochtones ${ }^{130}$. Le pays n'avait-il pas, en 1980 , porté à sa tête Julius Chan, dont le père était un immigré chinois ? Bien avant l'I-Kiribati Anote Tong en 2003, il fut le premier métis à accéder aux plus hautes fonctions d'un État océanien. Plus tard, le Papou- Néo-Guinéen d'origine chinoise Mao Zeming fut élu député et devint VicePremier ministre et ministre de la Défense ${ }^{131}$.

En avril 2006, suite à des élections législatives, une foule importante s'assembla devant le Parlement salomonais pour entendre la décision des députés, devant élire l'un des leurs comme premier ministre. L'annonce de l'élection de Snyder Rini, député sans étiquette, provoqua la colère des partisans de ses adversaires. Encouragée, selon des témoins, par des hommes politiques hostiles au nouveau dirigeant ${ }^{132}$, une partie de la foule se dirigea vers le quartier chinois de Honiara et "se mit à tout saccager ", brûlant notamment des magasins ${ }^{133}$. Soixante-quatorze bâtiments furent incendiés dans la capitale, dont cinquante-sept dans le quartier chinois. 317 personnes émigrèrent alors vers la Chine avec l'aide de l'Association chinoise, et de l'ambassade chinoise en Papouasie-NouvelleGuinée. Une commission gouvernementale sur les causes et les conséquences de l'émeute rapporta que certains hommes politiques avaient, pendant leur campagne, attisé la haine sinophobe des électeurs, l'exploitant dans une visée populiste. En période de difficulté économique, remarqua-t-elle, une minorité ethnique devenait une cible facile pour la rancœur populaire ; les commerçants chinois s'étaient ainsi vu accuser - sans preuve d'avoir corrompu des membres du gouvernement. Les incendies, poursuivit-elle, avaient clairement été déclenchés pour des raisons racistes, et avec "une indifférence inhumaine envers la vie humaine "; il aurait pu y avoir des morts ou des blessés graves ${ }^{134}$. Comme aux Tonga, un acte populaire de revendication politique s'était mué en violence sinophobe. Les immigrés chinois, naturalisés ou non, devenaient la cible de tous les ressentiments, dans un cadre d'instabilité politique et économique.

En 2007, le Taipei Times notait qu'environ 30000 immigrés asiatiques s'étaient installés en Papouasie-Nouvelle-Guinée au cours de la décennie précédente. Certains autochtones se plaignaient de les voir «contrôler » les petits commerces, ainsi que de la piètre qualité des produits que l'on y trouvait parfois. De tels ressentiments - rappelait le journal avaient généré des violences racistes aux Tonga et aux Îles Salomon ${ }^{135}$. La prévision s'avéra malheureusement exacte. Deux mois plus tard, les boutiques du quartier chinois 
de la ville de Mont Hagen furent dévalisées par des "gangs de pilleurs", et certaines incendiées ${ }^{136}$. En mai 2009, une vague d'agressions sinophobes se répandit en PapouasieNouvelle-Guinée. Elle suivit une confrontation entre travailleurs autochtones et responsables chinois d'une mine de nickel, provoquée par un accident du travail qui avait blessé un employé. Les autochtones protestaient également contre les dégâts environnementaux infligés par la mine à leurs terres. Trois ouvriers «chinois » avaient alors été « très gravement blessés " par leurs confrères indigènes ${ }^{137}$. Une « manifestation antichinoise", autorisée, se déroula à Port Moresby, rassemblant une centaine de personnes. Elle dégénéra en pillage de boutiques chinoises. Pendant plusieurs jours, des magasins chinois furent saccagés et des personnes d'origine asiatique agressées à Port Moresby, puis dans plusieurs autres villes, dont Mont Hagen à nouveau. Selon le Post Courier, les commerces de Mont Hagen étaient tenus à « $90 \%$ » par des « Asiatiques $»^{138}$. Des émeutiers expliquèrent qu'ils souhaitaient protester contre l'accroissement de l'immigration chinoise, leur « contrôle » des petits commerces, et les prix jugés excessifs de leurs produits - faisant écho aux prédictions, et aux événements de Nukualofa et de Honiara. Certains demandaient l'expulsion de tous les "Asiatiques " ${ }^{139}$. Les émeutiers, dans l'ensemble du pays, auraient été des « dizaines de milliers »; les affrontements avec les forces de l'ordre se soldèrent par quatre morts parmi les fauteurs de troubles ${ }^{140}$. Mekere Morauta, chef de l'Opposition et ancien Premier ministre, s'appuya sur les émeutes pour demander au gouvernement de restreindre l'immigration, afin d'empêcher des immigrés de venir « exploiter »le pays et ses habitants. Le gouvernement de Michael Somare, dit-il, avait permis à « des étrangers qui ne parlent pas un mot d'anglais de diriger des commerces que seuls les Papou-Néo-Guinéens devraient être autorisés à gérer » ${ }^{141}$. Les citoyens et leurs dirigeants découvraient la notion de priorité indigène, déjà développée aux Fidji. En novembre, un rapport des services de renseignement papou-néo-guinéens (National Intelligence Organisation) avertit que les sentiments anti-asiatiques demeuraient virulents, et que la possibilité de nouvelles violences était " potentiellement la menace la plus sérieuse envers la sécurité nationale du pays $»^{142}$. Le Vanuatu a été épargné par de tels phénomènes, malgré quelques actes d'agression isolés. Néanmoins, des ressentiments racistes se font parfois entendre. En 2005, un éditorial de l'Indépendant affirmait ainsi : « Au Vanuatu, les Chinois tiennent les commandes du commerce en particulier dans la capitale. Leur nombre croit de jour en jour. Ils risquent de nuire à la société [puisque], pour eux, le Vanuatu est l'endroit idéal pour s'enrichir, manipuler et tromper ses citoyens indigènes. La drogue et l'argent sale viendraient par la suite ${ }^{143}$. Ces propos haineux, dignes d'une certaine presse australienne du XIX siècle, ou d'une rhétorique populiste tongienne plus récente, font écho aux thèmes développés en Papouasie-Nouvelle-Guinée et aux Îles Salomon.

Les tensions entre Mélanésiens autochtones et citoyens mélanésiens d'origine chinoise ont toutefois une portée bien moins conséquente que celles qui opposent les Fidjiens indigènes à leurs compatriotes d'origine indienne. En mai 1987, le colonel Sitiveni Rabuka renversa le gouvernement du travailliste Timoci Bavadra, accusant ce Premier ministre autochtone d'avoir trop de ministres 'indiens'dans son gouvernement. S. Rabuka, qui se qualifiait lui-même d'homme "d'extrême droite " ${ }^{144}$, se fit le champion de la suprématie taukei (indigène). L'armée, composée presque exclusivement d'autochtones, le soutint sans hésitation. Il reçut en outre le soutien du Gouverneur-Général Ratu Penaia Ganilau qui dissout le Parlement -, de l'Église méthodiste - la principale Église chrétienne du pays -, et du Grand Conseil des Chefs. La crise révéla ainsi que les dirigeants des institutions les plus influentes étaient peu attachés à la démocratie. Un nouveau 'mouvement taukei', 
composé d'extrémistes nationalistes violents, soutint lui aussi le colonel, et orchestra des agressions physiques à l'encontre des biens et des personnes indo-fidjiens ${ }^{145}$.

Le coup d'État eut pour conséquence une nouvelle Constitution, restreignant la représentation politique des 'Indiens', contraignant chacun à ne voter que pour des candidats de sa propre 'race', et interdisant aux «Indiens » les plus hautes fonctions. Elle inscrivit dans les institutions nationales la suprématie politique de la "communauté » indigèn $\mathrm{e}^{146}$. Dans ce contexte, le pays connut une émigration « indienne » sans précédent. Des dizaines de milliers de citoyens des Fidji, d'origine indienne, fuirent l'archipel pour se réfugier en Australie et en Nouvelle-Zélande principalement. Constituant $48,7 \%$ de la population à la veille du coup d'État, les Indo-Fidjiens n'en comprenaient plus que 43,7\% en 1996, tandis que la proportion d'autochtones avait crû de $46 \%$ à 50,7\% ${ }^{147}$. Le coup d'État avait eu pour conséquence d'offrir aux «Fidjiens » une majorité non seulement relative mais absolue, pour la première fois depuis l'ère coloniale. Fidji n'était plus un pays majoritairement non-autochtone.

51 Les difficultés économiques des années qui suivirent, combinées aux pressions internationales, au climat de crise politique, et à l'éclatement du mouvement nationaliste en factions rivales, amenèrent à la rédaction d'une nouvelle constitution sept ans plus tard, avec l'accord des principaux partis politiques - et le soutien de Sitiveni Rabuka, qui s'était reconverti abruptement en défenseur du dialogue interculturel. La Constitution de 1997 révoqua l'obligation pour un premier ministre d'être indigène. Le préambule proclame : "Nous, le peuple des îles Fidji, [...] reconnaiss [ons] que les descendants de tous ceux qui ont choisi de vivre dans ces îles constituent notre société multiculturelle; affirm [ons] les contributions qu'apportent toutes ces communautés au bien-être de [notre] société $»^{148}$. Le thème d'unité dans la diversité recevait ainsi une officialisation constitutionnelle. Ce texte législatif suprême renomma en outre la République des Fidji en 'République des îles Fidji'( Republic of the Fiji Islands), ce qui permit enfin à tous ses habitants de se décrire comme Fiji Islanders (terme sans équivalent en français). Pour la première fois, les habitants de l'archipel recevaient une appellation nationale commune, censée promouvoir un sentiment d'unité. Le terme fut peu employé dans la langue courante, dans les médias ou par les hommes politiques.

52 En 1999, fut élu Mahendra Chaudhry, ancien leader syndicaliste, qui avait été ministre des finances sous Timoci Bavadra, et qui avait pris la tête du Parti travailliste. M. Chaudhry devint le premier Indo-Fidjien à accéder à la fonction de premier ministre. Un an plus tard, il fut renversé lors d'un coup d'État civil, mené par George Speight, ancien homme d'affaires en faillite, d'origine à la fois indigène et « européenne ». Adoptant l'ancienne rhétorique de Sitiveni Rabuka, il annonça qu'il était inadmissible qu'un Indo-Fidjien dirige le pays. À la tête d'un petit groupe de sept militants armés, il entra de force au Parlement et prit en otage pendant huit semaines le Premier ministre et plusieurs autres membres du gouvernement. Parallèlement, les partisans de G. Speight brûlèrent des écoles indo-fidjiennes et saccagèrent des temples hindous ${ }^{149}$. En conséquence, les "Indiens » continuèrent à émigrer par milliers. George Speight se rendit finalement à l'armée, et fut condamné à la prison à perpétuité pour haute trahison. Mais plutôt que de restituer Mahendra Chaudhry à ses fonctions, l'armée nomma un nouveau premier ministre par intérim - le banquier autochtone Laisenia Qarase, de tendance nationaliste. Il remporta les élections de 2001, et forma une coalition avec le parti fondé par les partisans de George Speight, l'Alliance conservatrice. Le gouvernement fut réélu en 2006. Il axa très explicitement sa politique sur la garantie de la suprématie des intérêts 
autochtones, et sur une politique de « discrimination positive » à leur égard. En 2003, le chef des armées, Voreqe Bainimarama, l'accusa d'appliquer la politique voulue par George Speight ${ }^{150}$. En 2005, le commodore accusa le premier ministre de racisme anti-indien, et le somma, en vain, de retirer les projets de loi discriminatoires, qu'il jugeait dangereux pour l'unité et l'harmonie du pays. Le 5 décembre 2006, V. Bainimarama et l'armée renversèrent le gouvernement. Pour la première fois, un coup d'État opposait l'armée, presque exclusivement autochtone, à un gouvernement autochtone nationaliste. Mais le contentieux concernait bien, avant tout, la question des relations entre les deux principaux groupes ethniques des Îles Fidji, et leurs places respectives dans la société.

Dirigeant un gouvernement « de transition », Voreqe Bainimarama demeure au pouvoir à ce jour, ayant muselé la presse, abrogé la Constitution, et promis des élections démocratiques en septembre 2014, suite à l'application de réformes visant à inhiber par avance toute politique « raciste » de la part d'un gouvernement élu. Son objectif annoncé est de "bâtir la nation" (nation-building) ${ }^{151}$. Une nouvelle Constitution en 2013 - la quatrième dans l'histoire du pays - doit abolir les listes électorales ethniques. Chaque voix aurait la même valeur, et les députés représenteraient les citoyens dans leur ensemble, plutôt qu'une communauté ethnique. Par ailleurs, le nom du pays cesserait d'être Fiji Islands, et redeviendrait Fiji. Ceci permettrait à tous les citoyens de s'appeler «Fidjiens». Le terme ne serait plus réservé aux autochtones; il deviendrait une appellation nationale, et non pas ethnique. Les écoles enseigneraient les diverses langues du pays, ainsi que les fondements de ses différentes religions. Plus généralement, l'enseignement devrait promouvoir le multiculturalisme. Le gouvernement cesserait d'étiqueter les citoyens selon leur « race », sauf dans la perspective de préserver les droits fonciers spécifiques aux autochtones. Enfin, les autorités promouvraient "une vision morale, des récits, des rituels et des symboles nationaux "152. Une "cérémonie du drapeau " serait instaurée dans toutes les écoles. Un patriotisme pluri-ethnique serait ainsi forgé par le gouvernement, mobilisant les principaux acteurs de la société. Les ressentis identitaires seraient, dans la mesure du possible, altérés par l'action « pédagogique » de l'État.

Ainsi, de manière inquiétante, certains Mélanésiens revendiquent pour identité nationale une unité "raciale» face à des communautés d'origine immigrée, principalement asiatiques. Une reconnaissance ethnique serait apte à souder les autochtones, à travers l'exclusion des «autres", et à l'image d'identités nationales « raciales » longtemps prônées en Nouvelle-Zélande et en Australie. Les dirigeants mélanésiens actuels rejettent ces polarisations fondées sur la méfiance et la haine, mais ne parviennent pas encore trois à quatre décennies après l'indépendance - à fédérer leurs citoyens autour d'un sentiment d'appartenance plus constructif. L'ensemble des États océaniens a dépassé le stade de la promotion d'une identité «raciale » ou monoethnique - bien qu'aux Fidji ce dépassement soit principalement la conséquence d'un coup d'État-, mais une minorité conséquente de citoyens demeure attachée à des identités "raciales » exclusives, en apparence plus simples et mieux enracinées.

Pendant l'ère impériale, l'Australie et la Nouvelle-Zélande s'auto-définissaient comme des terres réservées la « race » blanche, sur le fondement d'un consensus politique durable. Cet objectif fut mis en application par la fermeture des frontières selon des critères « raciaux», et par l'assimilation des autochtones (en Nouvelle-Zélande), ou leur exclusion et leur ségrégation (en Australie). Simultanément, dans les îles du Pacifique, les autorités britanniques mettaient en œuvre une politique de "préservation» des 
populations indigènes par le biais d'un respect et d'une promotion de certains aspects au moins de leur mode de vie; par le biais également de la ségrégation; et, parfois notamment dans les îles sous administration australienne ou néo-zélandaise -, en restreignant la venue de migrants asiatiques, ou leurs rapports avec les autochtones. À partir des années 1960, l'Australie et la Nouvelle-Zélande démantelèrent leurs politiques de discrimination 'raciale', tandis que l'Empire se retirait petit à petit de ses microterritoires du Pacifique. À l'heure où Canberra et Wellington proclamaient soudain la promotion de la diversité culturelle et ethnique, dans les années 1970, les nouveaux États indépendants de la région envisageaient leurs propres politiques identitaires. À quelques exceptions près, ils fondèrent leurs affirmations d'identité nationale sur les «traditions »- nécessairement autochtones - tout en garantissant aux citoyens nonindigènes l'égalité des droits civiques et politiques. Le multiculturalisme officiel intégrant les minorités d'origine immigrée (en Australie, aux Fidji et en Nouvelle-Zélande), ou simplement l'acceptation par les autorités de la diversité ethnique, ne sont toutefois pas toujours en adéquation avec la revendication d'une primauté blanche ou autochtone exprimée par une minorité conséquente - et parfois violente - de la population.

\section{NOTES}

1. Toon VAN MEIJL, "The New Zealand Wars and the Maori King Movement", in Donald DENOON, (éd.), The Cambridge History of the Pacific Islanders, Cambridge: Cambridge University Press, 1997, pp. 165-6.

2. Geoffrey BLAINEY, “Australia Unlimited”, Boyer Lectures, Radio National, 11 novembre 2001, [ http://www.abc.net.au/radionational/programs/boyerlectures/lecture-1-australiaunlimited/3501650\#transcript]

3. James JUPP, The English in Australia, Cambridge: Cambridge University Press, 2004, pp. 68-9.

4. Michael KING, The Penguin History of New Zealand, Auckland: Penguin Books, 2003, p. 229.

5. George GREY, discours à la Chambre des Représentants, 1879, cité in Nigel MURPHY, "Joe Lum v. The Attorney General: The Politics of Exclusion", in IP, Manying (éd.), Unfolding History, Evolving Identity: The Chinese in New Zealand, Auckland : Auckland University Press, 2003, p. 49.

6. "a home fit for the best of the Anglo-Saxon race - a White Man's country". William MASSEY, "A White Man's Country”, Empire Mail, vol. 13, n 10, oct-nov 1924, p. 587.

7. Keith MURDOCH, “Australia Day : 133 years of progress”, The Times, 26 janvier 1921, p. 11.

8. M.L SHEPHERD, “White Australia: An Enduring Ideal”, lettre à l'éditeur du Times, 7 janvier 1922, p. 6.

9. Alfred DEAKIN, discours devant le Parlement fédéral, 12 septembre 1901, cité in KELLY, Paul, The End of Certainty: Power, Politics \& Business in Australia, St Leonards : Allen \& Unwin, 1994, p. 3.

10. Vince MAROTTA, "The ambivalence of borders: the bicultural and the multicultural”, in John DOCKER \& Gerhard FISCHER, (éds.), Race, Colour \& Identity in Australia and New Zealand, Sydney: University of New South Wales Press, 2000, p. 180.

11. Anon., "Wellington's Chinatown: Plague Spots of Asiatic Vice in Our Midst", New Zealand Times , 30 juin 1896, p. 2. 
12. Brian MOLOUGHNEY, Tony BALLANTYNE, \& David HOOD, “After Gold: Reconstructing Chinese Communities, 1896-1913", in Henry JOHNSON \& Brian MOLOUGHNEY, Asia in the Making of New Zealand, Auckland: Auckland University Press, 2006, pp. 72-4.

13. Francine TOLRON, La Nouvelle-Zélande: du duel au duo?, Toulouse : Presses Universitaires du Mirail, 2000, p. 210.

14. Anon., rapport accompagnant les données du recensement de 1921, 6 ème partie: "Race aliens", p. 1.

15. James JUPP, The English in Australia, op.cit., pp. 120-1.

16. James JUPP, From White Australia to Woomera, Cambridge: Cambridge University Press, 2002, p. 9.

17. James BELICH, Making Peoples: A History of the New Zealanders from Polynesian Settlement to the End of the Nineteenth Century, Honolulu : University of Hawai'i Press, 1996, p. 315.

18. Gouvernement du Queensland, “The Australian South Sea Islander community", p. 6, [http:// www.premiers.qld.gov.au]

19. Brian MURPHY, The Other Australia: Experiences of Migration, Cambridge : Cambridge University Press, 1993, p. 23.

20. Ien ANG, "Asians in Australia : a contradiction in terms?", in John DOCKER, \& Gerhard FISCHER, (éds.), Race, Colour \& Identity in Australia and New Zealand, op.cit., p. 126.

21. Francine TOLRON, La Nouvelle-Zélande : du duel au duo ?, op.cit., p. 130.

22. Geoffrey BLAINEY, The Tyranny of Distance, Melbourne: Sun Books, 1980 [1966], p. 203.

23. Bessie NG KUMLIN ALI,, Chinese in Fiji, Suva : University of the South Pacific, 2002, p. 29.

24. Circulaire du gouvernement aux autorités douanières, Home \& Territories Department, 4 mars 1927, reproduite in La Trobe University, Asian Studies Program, "Example of material held in the Boarding Branch Circulars detailing how the Dictation Test should be applied", Digital Documents Record, [http://www.latrobe.edu.au]

25. Anon., cité in Anon., "Labour Questions in Australia: 'A White Australia”, The Times, 3 septembre 1902, p. 6.

26. Anon., "Australia in the Pacific: The beginning of direct representation relations with the 'Near North", The Times, 6 septembre 1941, p. 5.

27. Donald DENOON, "New Economic Orders: Land, Labour and Dependency", in Donald DENOON, (éd.), The Cambridge History of the Pacific Islanders, op.cit., p. 244.

28. Traité de 1840 signé par des chefs maori et des représentants de la Couronne, permettant au Royaume-Uni d'annexer la Nouvelle-Zélande en contrepartie de la reconnaissance de certains droits aux autochtones.

29. Frank WELSH, Great Southern Land: A New History of Australia, Londres : Penguin, 2005, p.xxxiv.

30. Geoffrey BLAINEY, A Land Half Won, Melbourne: Sun Books, 1987, p. 93.

31. Peter BISKUP, Not Slaves Not Citizens, St Lucia: University of Queensland Press, 1979, p. 154.

32. George W. RUSDEN, History of New Zealand, Melbourne: Chapman \& Hall, Ltd., 1883, p. 375.

33. James BELICH, Paradise Reforged: A History of the New Zealanders from the 1880s to the Year 2000, Honolulu: University of Hawai'i Press, 2001, p. 189.

34. William MASSEY,cité in Anon., "Banning the undesirable: Immigration Restriction Bill", Auckland Star, 15 septembre 1920, p. 8.

35. Myrna E. TONKINSON, "Is It in the Blood? Australian Aboriginal Identity", in Jocelyn LINNEKIN \& Lin POYER, Cultural Identity and Ethnicity in the Pacific, Honolulu: University of Hawai' $\mathrm{i}$ Press, 1990, p. 203.

36. Le terme renvoie à une forme de camaraderie fondée sur l'entraide, fréquemment invoquée, du XIXe siècle à aujourd'hui, comme une valeur australienne primordiale.

37. Amendement de la loi Commonwealth Electoral Act de 1918.

38. Phillip KNIGHTLEY, Australia: a biography of a nation, Londres: Vintage, 2001, p. 261. 
39. Ien ANG, "Asians in Australia: a contradiction in terms?", in John DOCKER \& Gerhard FISCHER, (éds.), Race, Colour \& Identity in Australia and New Zealand, op.cit., p. 121.

40. Al GRASSBY, A Multi-Cultural Society for the Future, Canberra: Australian Government Publishing Service, 1973.

41. Anon., "Multicultural laws could harm biculturalism - Carter", Radio New Zealand, 28 juin 2008, [http://www.radionz.co.nz].

42. Arthur GORDON, cité in Margaret JOLLY, "Custom and the Way of the Land: Past and Present in Vanuatu and Fiji", in Robert BOROFSKY, (éd.), Remembrance of Pacific Pasts: An Invitation to Remake History, Honolulu: University of Hawai'i Press, 2000, p. 348.

43. Peter MITCHELL, cite in Stewart FIRTH, "Colonial Administration and the Invention of the Native", in Donald DENOON, (éd.), The Cambridge History of the Pacific Islanders, op.cit., p. 268.

44. Brij V. LAL, "The passage out", in K.R., HOWE, Robert KISTE \& Brij LAL,Tides of History: The Pacific Islands in the Twentieth Century, Honolulu : University of Hawai'i Press, 1994, p. 405.

45. Brij V. LAL, Islands of Turmoil: Elections and Politics in Fiji, Canberra: Australian National University, Asia Pacific Press, 2006, p. 3.

46. A.A. RAGG, 1946, cité in John D. KELLY \& Martha KAPLAN, Represented Communities: Fiji and World Decolonization, Chicago : University of Chicago Press, 2001, p. 169.

47. Barrie MACDONALD, Cinderellas of the Empire: Towards a history of Kiribati and Tuvalu, Suva : University of the South Pacific, 2001, p. 137.

48. Judith BENNETT, "Holland, Britain, and Germany in Melanesia", in K.R. HOWE, Robert KISTE, \& Brij LAL, Tides of History, op.cit., p. 57.

49. Bessie NG KUMLIN ALI, Chinese in Fiji, op.cit., pp. 56-7.

50. H. Ian HOGBIN, Experiments in Civilization: The Effects of European Culture on a Native Community of the Solomon Islands, New York: Schocken Books, 1970 [1939], p. 242.

51. Bill WILLMOTT, "Early History of the Chinese in Vanuatu: 1844-1944", in Brian MOLOUGHNEY

\& Jim NG, (éds.), Chinese in Australasia and the Pacific: Old and New Migrations and Cultural Change, Dunedin: University of Otago, 1998, pp. 244-246.

52. Pierre Yves TOULLELAN \& Bernard GILLE, De la Conquête à l'Exode: Histoire des Océaniens et de leurs migrations dans le Pacifique, Tahiti : Au Vent des Îles, 1999, p. 181.

53. Ibid, p. 178.

54. Donald DENOON, "New Economic Orders: Land, Labour and Dependency", in Donald DENOON, (éd.), The Cambridge History of the Pacific Islanders, op.cit., p. 160.

55. Pierre Yves TOULLELAN \& Bernard GILLE, De la Conquête à l'Exode, op.cit., p. 177.

56. George RICHARDSON, "That Yellow Taint", New Zealand Times, 28 février 1923, cité in F.Ben LIUA'ANA, "Dragons in Little Paradise: Chinese (Mis-)Fortunes in Samoa, 1900-1950", The Journal of Pacific History, vol. 32, n 1, 1997, p. 34.

57. Joeli BALEDROKADROKA, "The Fijian Understanding of the Deed of Cession Treaty of 1874", Indiana University Digital Library, 2003, p. 52, [http://dlc.dlib.indiana.edu].

58. Pierre Yves TOULLELAN \& Bernard GILLE, De la Conquête à l'Exode, op.cit., p. 178.

59. Maynard HEDSTROM, "Fiji Today: Fifty Years of British Rule", The Times, 10 octobre 1924, p. 11.

60. Élise HUFFER, Grands hommes et petites îles : la politique extérieure de Fidji, de Tonga et du Vanuatu, Paris : Orstom, 1993, pp. 22-3.

61. John D. KELLY \& Martha KAPLAN, Represented Communities, op.cit., p. 130.

62. Ibid, p. 169.

63. La population de Nauru en 1966 était de 6 048, dont seulement 2921 autochtones. Nancy J. POLLOCK, "Nauru's Post-Independence Struggles", in Brij V. LAL \& Hank NELSON, (éds.), Lines Across the Sea : Colonial Inheritance in the Post Colonial Pacific, Brisbane : Pacific History Association, 1995, p. 53.

64. Département d'État américain, "Background Note : Nauru”, [http://www.state.gov]. 
65. Bill WILLMOTT, The Chinese Communities in the Smaller Countries of the South Pacific: Kiribati, Nauru, Tonga and the Cook Islands, Christchurch: Macmillan Brown Centre for Pacific Studies, 2007, p. 35.

66. Electoral Amendment Act, 1990.

67. Uentabo NEEMIA, "Decolonization and Democracy in the South Pacific", in Ron CROCOMBE, Uentabo NEEMIA, Asesela RAVUVU \& Werner VOM BUSCH, (éds.), Culture \& Democracy in the South Pacific, Suva : University of the South Pacific, 1992, p. 8.

68. Aigaletaule'ale'à TAUAFIAFI, "Samoan elections : Tuilaepa perches on the cusp of history", Pacific Media Centre, 3 mars 2011, [http://pacific.scoop.co.nz].

69. Parlement des Samoa, "Members of Parliament 2011 - 2016", [http:// www.parliament.gov.ws].

70. John D. KELLY \& KAPLAN, Martha, Represented Communities, op.cit., pp. 130-3.

71. Jai Ram REDDY, in Constitutional Review Commission: Verbal Transcripts of Hearings, vol. 3,24 août 1995, p. 5.

72. Vice-Président de la République des Îles Fidji de 2004 à 2006.

73. Joni MADRAIWIWI, “Governance in Fiji : The Interplay between Indigenous Tradition, Culture and Politics", in Stewart FIRTH, (éd.), Globalisation and Governance in the Pacific Islands, Canberra : Australian National University, 2006, p. 294.

74. Vijay NAIDU, "The State of the State in Fiji: Some Failings in the Periphery", in Ibid, p. 297.

75. Ministère des Affaires fidjiennes et de la Culture, [http://www.culture.gov.fj].

76. La plupart des Constitutions des États océaniens déclarent que la nation est fondée sur les coutumes et les traditions autochtones, ancestrales.

77. Vanuatu Cultural Centre, [http://www.vanuatuculture.org].

78. Ministère de l'Éducation, des Sports et de la Culture, Social Studies Years 9-11: Sāmoa Secondary School Curriculum, janvier 2004, [http://www.mesc.gov.ws].

79. Michael SOMARE, discours prononcé le 16 septembre 2006, [http://www.pm.gov.pg].

80. Ann CURTHOYS, "An uneasy conversation : the multicultural and the indigenous", in John DOCKER \& Gerhard FISCHER, (éds.), Race, Colour \& Identity in Australia and New Zealand, op.cit., p. 28.

81. Ien ANG, “Asians in Australia: a contradiction in terms?”, in Ibid, p. 124.

82. James JUPP, From White Australia to Woomera, op.cit., p. 56.

83. Fran KELLY, "Tampa issue improves Coalition election prospects", Australian Broadcasting Corporation, 4 septembre 2001, [http://www.abc.net.au].

84. Philippa MEIN SMITH, A Concise History of New Zealand, Cambridge : Cambridge University Press, 2005, p. 246.

85. Sekhar BANDYOPADHYAY, "Reinventing Indian Identity in Multicultural New Zealand", in Henry JOHNSON \& Brian MOLOUGHNEY, Asia in the Making of New Zealand, op.cit., p. 136.

86. Cette accusation est démentie par les études démographiques.

87. Pauline HANSON, discours prononcé le 10 septembre 1996, [http://nswonenation.com.au].

88. James JUPP, From White Australia to Woomera, op.cit., p. 135.

89. Jared SAVAGE \& Leah HAINES, "Winston Peters' last stand is a lost battle", New Zealand Herald, 9 novembre 2008, [http://www.nzherald.co.nz].

90. Australian Bureau of Statistics, "Religious Affiliation", [http://www.abs.gov.au].

91. Kim CAMBERG, "Long-term tensions behind Sydney riots", British Broadcasting Corporation, 13 décembre 2005, [http://news.bbc.co.uk].

92. Francene NORTON, “Danna Vale recycles ‘populate or perish' politics”, Australian Broadcasting Corporation, 14 février 2006.

93. Anon., "Bar Africans with 'diseases', Hanson says", Daily Telegraph, 6 décembre 2006, [http:// www.news. com.au/dailytelegraph]. 
94. Nick BRYANT, "Surf's up for Australian Muslims", British Broadcasting Corporation, 2 février 2007.

95. Anon., cité in Nick BRYANT, "Town moves against Islamic school", British Broadcasting Corporation, 26 mai 2008.

96. Anon., "One in 10 Tasmanians racist", The Mercury, 29 septembre 2008, [http:// www.news.com.au/mercury].

97. Tonga Department of Statistics, "Population Census 2006: Social characteristics", [http:// www.spc.int].

98. Département d'État américain, "Background Note : Samoa", [http://www.state.gov].

99. Kiribati National Statistics Office, "Population by Ethnic Group and Island - 2005", [http:// www.spc.int].

100. Programme des Nations unies pour le Développement, “Tuvalu”, [http://www.undp.org.fj].

101. A. Morgan TUIMALEALI'IFANO, "Matai Titles and Modern Corruption in Samoa: Costs, expectations and consequences for families and society", in FIRTH, Stewart (éd.), Globalisation and Governance in the Pacific Islands, op.cit.,

102. Anon., "Land Reform Bill not Samoan, says Samoa Party", Radio New Zealand International, 30 avril 2008, [http://www.rnzi.com].

103. Anon., "Étude de faisabilité pour une Commission des droits de l'Homme au Samoa ", $A B C$ Radio Australia (Brèves du Pacifique), 6 avril 2009, [http://24hdanslepacifique.com].

104. Anon., "Samoa MP accused of racism over anti-Chinese remark", Radio New Zealand International, 21 janvier 2005.

105. Batiri BATAUA, "Burning of Truck In Tarawa A Sign of Escalating Anti-Chinese Sentiment ?", Pacific Magazine, 20 septembre 2008, [http://www.pacificmagazine.net].

106. Anon., "Kiribati committee to probe immigration approvals", Radio New Zealand International, 7 juin 2009.

107. Anon., "Tong re-elected Kiribati president”, ABC Radio Australia, 18 octobre 2007.

108. Anon., "The Chinese and Indians will take over: Stop it now or it will be too late", Ko e Kelea, vol. 7, n 3, juin 1992, p. 1.

109. John HENDERSON, "China, Taiwan and the Changing Strategic Significance of Oceania", Revue Juridique Polynesienne, hors série $\mathrm{n}^{\circ}$ 1, 2001, p. 157.

110. Anon., "Chinese in Tonga", Matangi Tonga, 17 mars 2006, [http://www.matangitonga.to].

111. Anon., "No More Chinese!", Tongatapu.net, 19 août 2000, [http://www.tongatapu.net.to].

112. Paul RAFFAELE \& Matthew DEARNALEY, “Tonga to expel race-hate victims", New Zealand Herald, 22 novembre 2001.

113. Anon., "Editorial: Racist moves will rebound on Tonga", New Zealand Herald, 23 novembre 2001.

114. Anon., "Pro-democracy supporters and youth take to the streets of Nuku'alofa, damaging and looting government and business offices", Tonga Broadcasting Corporation, 16 novembre 2006, [ http://www.tonga-broadcasting.com].

115. Anon., "People of Havelu apologise to Chinese shop owner", Tonga Broadcasting Corporation, 14 décembre 2006.

116. Anon., "Tonga's king vows more democracy", British Broadcasting Corporation, 23 novembre 2006.

117. Sujets de discussion: "Why Targeting the Chinese ?" [sic] ; "Leave the Chinese and Indians alone - They contribute!!!", Planet Tonga, novembre 2006, [http://www.planet-tonga.com/ forums].

118. Fiji Islands Bureau of Statistics, recensement de 2007, [http://www.statsfiji.gov.fj].

119. Ces hommes furent tous nommés sénateurs par des gouvernements de tendance nationaliste autochtone. Bessie NG KUMLIN ALI, Chinese in Fiji, op.cit., pp. 195, 211. 
120. Margaret JOLLY, "Custom and the Way of the Land: Past and Present in Vanuatu and Fiji", in Robert BOROFSKY (éd.), Remembrance of Pacific Pasts, op.cit., p. 354.

121. Michael ONG, "Fiji: May Elections and the New Government", Current Issues Brief, $n^{\circ} 17$, Parliamentary Library (Australie), 29 juin 1999, [http://www.aph.gov.au].

122. Vanuatu Statistics Office, "Vanuatu Population Summary", [http:// www.vanuatustatistics.gov.vu].

123. Ambassade de la République populaire de Chine au Vanuatu, "Chinese in Vanuatu", [http:// www.vanuatu embassy.org.cn].

124. Bill WILLMOTT, "Early History of the Chinese in Vanuatu: 1844-1944", in Brian MOLOUGHNEY \& Jim NG, (éds.), Chinese in Australasia and the Pacific, op.cit., p. 241.

125. Commonwealth des Nations, "Papua New Guinea- Society", 2008, [http:// www.thecommonwealth.org].

126. Commonwealth des Nations, "Solomon Islands - Society", 2008.

127. Paul SILLITOE, Social Change in Melanesia: Development and History, Cambridge: Cambridge University Press, 2000, p. 15.

128. Parlement des Îles Salomon, "Members of the Sixth Parliament", [http:// www.parliament.gov.sb].

129. Honiara se situe à Guadalcanal, où s'installent de nombreux migrants internes venus d'autres provinces.

130. John D.WAIKO, A Short History of Papua New Guinea, Melbourne : Oxford University Press, 1993, p. 251.

131. Joe R. KANEKANE, "Governance, Globalisation and the PNG Media : A survival dilemma”, in Stewart FIRTH, (éd.), Globalisation and Governance in the Pacific Islands, op.cit..

132. Rapport de la Commission d'enquête sur les troubles civils à Honiara en avril 2006 ( Commission of Inquiry into the April 2006 Civil Unrest in Honiara), publié dans le Solomon Star, 20 septembre 2007, [http://www.solomonstarnews.com].

133. Anon., "Pacific Almanac 2007 : Solomon Islands", Pacific Magazine.

134. Rapport de la Commission d'enquête sur les troubles civils à Honiara en avril 2006, op.cit.

135. Anon., "China's fuel drive draws ire in Papua New Guinea”, Taipei Times, 9 juillet 2007, [ http://www.taipeitimes.com].

136. Phil MERCER, "Chinese targeted by PNG rioters", British Broadcasting Corporation, 21 septembre 2007.

137. Ilya GRIDNEFF, “More attacks at PNG's Chinese run mine”, The Age, 11 mai 2009, [http:// news.theage.com.au].

138. Robert PALME, “Total mayhem”, Post Courier, 20 mai 2009, [http://www.postcourier.com.pg]. 139. Pisai GUMAR, "Asian shops hit in Lae", The National, 15 mai 2009, [http:// www.thenational.com.pg].

140. Rowan CALLICK, "Looters shot dead amid chaos of Papua New Guinea's anti-Chinese riots", The Australian, 23 mai 2009.

141. Mekere MORAUTA, interrogé par ROBERTS, Greg, "Papua New Guinea [sic] vow to burn all Asian shops", The Australian, 29 mai 2009.

142. Liam FOX, "PNG intelligence agency warns of ethnic tensions", Australia Network News, 6 novembre 2009, [http://australianetworknews.com].

143. Gratien TIONA, «Éditorial : Quel sera le sort de Ham Lini avec les Chinois? ", L'Indépendant, 7 mars 2005, [http://www.news.vu].

144. Sitiveni RABUKA, août 1990, cité in LAL, Brij V., Islands of Turmoil, op.cit., p. 105.

145. Sanjay RAMESH, “The Fijian Military's 'Clean Up”, World Press, 2 février 2007, [http:// www.worldpress.org].

146. Constitution of the Sovereign Democratic Republic of Fiji, 1990. 
147. Joeli BALEDROKADROKA, “The Fijian Understanding of the Deed of Cession Treaty of 1874", Indiana University Digital Library, op.cit., p. 52.

148. Constitution (Amendment) Act, 1997.

149. Brij V. LAL, Islands of Turmoil, op.cit., p. 188.

150. Sanjay RAMESH, “The Fijian Military's “Clean Up”, World Press, op.cit.

151. Anon., "Fiji interim PM restates constitution will be in place for 2014 polls", Radio New Zealand International, 21 juillet 2009.

152. National Council for Building a Better Fiji, Peoples [sic] Charter for Change, Peace and Progress, Suva, 15 décembre 2008, p. 18.

\section{RÉSUMÉS}

La tentation de fonder une affirmation identitaire nationale sur une adéquation entre 'race' et nation a marqué les discours et les politiques en Océanie, à l'instar d'autres régions du monde. Cet article se donne pour objectif d'analyser la manière dont l'impératif d'unité nationale s'y est appuyé sur la perception ou la mise en avant d'une homogénéité non seulement culturelle mais ethnique ou "raciale», accompagnée parfois de politiques d'exclusion des minorités. En Australie et en Nouvelle-Zélande, la politique actuelle de promotion de la diversité culturelle fait suite à une longue période d'accord bipartite sur la préservation de nations exclusivement «blanches » et «britanniques ». Dans les îles du Pacifique, les politiques de discrimination sont rares depuis l'indépendance - malgré des contre-exemples aux Fidji et à Nauru, pays dotés d'importantes minorités d'origine étrangère-, mais l'image que se donnent ces nations repose sur une homogénéité ethnoculturelle autochtone, où les minorités paraissent toujours «étrangères ». En outre, des violences sinophobes, fruit d'un rejet de minorités chinoises trop visibles, ont frappé ces dernières années plusieurs pays insulaires, comme en écho à des violences similaires qui ont marqué autrefois l'Australie. Cette étude s'intéresse à la place accordée aux minorités dans des pays du Pacifique partagés entre des nations sûres de leur identité homogène, et celles tentant de bâtir une identité nationale multiculturelle encore incertaine.

Discourses and politics in the Pacific, as in other regions of the world, have at times equated 'race' and nation as a basis for statements on national identity. The objective of this article is to analyse the ways in which the requirement for national unity has been grounded, in that region, on the perception or promotion not just of a cultural homogeneity, but also an ethnic or 'racial' one, sometimes reinforced by policies to exclude minorities. In Australia and New Zealand, the current promotion of cultural diversity by the governments contrasts with a long-lasting, earlier bipartisan agreement on the preservation of exclusively 'White' and 'British' nations. In the Pacific islands, state-endorsed discrimination has been rare since independence -despite counter-examples in Fiji and Nauru, two countries with sizeable minorities of foreign descent-, but the self-image of these nations rests upon that of a homogenous indigenous ethnicity and culture, wherein minorities appear permanently 'foreign'. In addition, persons of Chinese origin, part of an increasingly visible minority, have been subject to rejection, and even to racially motivated violence, in several island countries in recent years - as though in echo to a similar violence which once marked Australia. This study focuses on the place granted to minorities in 
Pacific countries, divided between nations certain of their homogenous identity, and those attempting to build a still uncertain form of multicultural national identity.

\section{AUTEUR}

\section{ADRIEN RODD}

Adrien Rodd est maître de conférences en civilisation britannique à l'Université de Versailles-St Quentin, membre du laboratoire Suds d'Amériques. Ancien élève de l'École Normale Supérieure de Cachan et agrégé d'anglais, il a soutenu en 2010 une thèse intitulée « Constructions identitaires nationales et britannicité dans les pays du Commonwealth en Océanie ». Son centre d'intérêt principal en recherche porte sur les problématiques identitaires dans les anciennes colonies britanniques. Il a à son actif plusieurs articles et communications dans ce champ d'études. 